DAMTP-2001-103

\title{
Dual variables and a connection picture for the Euclidean Barrett-Crane model
}

\author{
Hendryk Pfeiffer* \\ Emmanuel College, St. Andrew's Street, Cambridge CB2 3AP \\ and \\ Department of Applied Mathematics and Theoretical Physics, \\ Wilberforce Road, Cambridge CB3 OWA \\ England, UK
}

30 January 2002

\begin{abstract}
The partition function of the $S O(4)$ - or Spin(4)-symmetric Euclidean Barrett-Crane model can be understood as a sum over all quantized geometries of a given triangulation of a four-manifold. In the original formulation, the variables of the model are balanced representations of $S O(4)$ which describe the quantized areas of the triangles. We present an exact duality transformation for the full quantum theory and reformulate the model in terms of new variables which can be understood as variables conjugate to the quantized areas. The new variables are pairs of $S^{3}$-values associated to the tetrahedra. These $S^{3}$ variables parameterize the hyperplanes spanned by the tetrahedra (locally embedded in $\mathbb{R}^{4}$ ), and the fact that there is a pair of variables for each tetrahedron can be viewed as a consequence of an $S O(4)$-valued parallel transport along the edges dual to the tetrahedra. We reconstruct the parallel transport of which only the action of $S O(4)$ on $S^{3}$ is physically relevant and rewrite the Barrett-Crane model as an $S O(4)$ lattice $B F$-theory living on the 2-complex dual to the triangulation subject to suitable constraints whose form we derive at the quantum level. Our reformulation of the Barrett-Crane model in terms of continuous variables is suitable for the application of various analytical and numerical techniques familiar from Statistical Mechanics.
\end{abstract}

PACS: $04.60 . \mathrm{Nc}$

key words: Spin foam model, Barrett-Crane model, quantum gravity, duality transformation

\section{Introduction}

Spin foam models have been proposed as candidates for a quantum theory of space-time geometry [1 5] and therefore form candidates for a quantum theory of gravity. Spin foam models also play a central role in the construction of invariants of piecewise-linear three- and

*e-mail: H.Pfeiffer@damtp.cam.ac.uk 
four-manifolds [6, 7]. They arise as the models that are strong-weak dual to non-Abelian lattice gauge theory [8, 9] and form a possible starting point for the generalization of gauge theories from Lie groups to quantum groups [10, 11]. For reviews on the subject, see, for example [12, 13].

A spin foam [5] whose symmetry group is a compact Lie group $G$, is an abstract oriented 2-complex consisting of faces, edges and vertices, together with a colouring of the faces with representations of $G$ and a colouring of the edges with compatible intertwiners (representation morphisms) of $G$. A spin foam model is a discrete model in the framework of Statistical Mechanics whose partition function is a sum over spin foams each of which is assigned a Boltzmann weight. If the Boltzmann weight factors into contributions for each vertex, edge and face, these factors are usually called vertex, edge and face amplitudes.

Barrett, Crane and Baez [1, 5, 14] have developed a particular spin foam model whose partition function can be understood as the sum over all quantized Euclidean geometries that can be assigned to a given triangulation of a four-manifold. The spin foam model is usually defined on the 2-complex dual to that triangulation. Several versions of this model are studied in the literature [15] 17] which differ in their Boltzmann weights (amplitudes), in particular in their edge amplitudes. Some of the models are formulated on a fixed 2-complex while others include a sum over 2-complexes. In the following, we call all these models Barrett-Crane models.

In this article, we study a generalized version which encompasses all interesting choices of amplitudes, but we restrict ourselves to a fixed 2-complex. The partition function of our model is a sum over all possible assignments of balanced irreducible representations of the Lie group $S O(4)$ to the faces. The balanced representations are of the form $V \otimes V$ where $V$ is an irreducible representation of $S U(2)$. They are also called the simple representations. Each edge is then assigned a unique intertwiner, the Barrett-Crane intertwiner [ $\llbracket$. We allow quite generic edge and face amplitudes, but the vertex amplitude is always the generalized $10 j$-symbol [4, [5] consisting of one Barrett-Crane intertwiner for each edge attached to the vertex.

We make use of the fact that the Barrett-Crane intertwiner can be written in terms of an integral over the sphere $S^{3}$ [18, 19] and of the techniques familiar from the duality transformation for non-Abelian lattice gauge theory [8, 10] and derive a dual expression for the partition function of the Barrett-Crane model in which the fundamental variables are pairs of $S^{3}$-values associated to the edges. The partition sum is then given by an integral over $S^{3}$ for each of these variables.

Provided that the edge amplitude of the original model is of a particular form, the dual model has local interactions, its Boltzmann weight factors into one contribution for each face, and it depends on the $S^{3}$-variables at the edges in the boundary of that face.

This reformulation of the Barrett-Crane model has the following geometric interpretation if the 2-complex is chosen to be dual to a triangulation of a four-manifold. The $S^{3}$-variables are then associated to the tetrahedra. They parameterize the (normalized) normal vectors of the hyperplanes spanned by the tetrahedra where the tetrahedra are locally embedded in $\mathbb{R}^{4}$. The factorized Boltzmann weight provides an interaction term for each triangle so that the interaction depends on the $S^{3}$-values associated to all tetrahedra that contain the triangle in their boundaries.

However, there are two $S^{3}$-values associated to each tetrahedron one of which belongs to either one of the two four-simplices that contain the tetrahedron in their boundaries. This suggests that there exists an $S O(4)$-valued parallel transport along the tetrahedron which 
maps the first $S^{3}$-value to the second one. However, this parallel transport is defined only up to elements of $S O(4)$ that stabilize the first of the $S^{3}$-values. Following this idea, we show that the Barrett-Crane model can be obtained from $S O(4)$ lattice $B F$-theory (the $S O(4)$ Ooguri model [20, 21]) if we impose a constraint at the quantum level by averaging over this stabilizer in a suitable way. This means that of the $S O(4)$-valued parallel transport only the action of $S O(4)$ on $S^{3}$ is physically relevant.

Finally, we consider the Boltzmann weight of the dual formulation of the Barrett-Crane model and list possible face and edge amplitudes for which the partition function is well defined (not just divergent). We outline how one can analyze this model in the context of Statistical Mechanics, for example in order to study its ground state and fluctuations around it. In addition, one can expect that the dual formulation of the Barrett-Crane model is well suited for numerical studies.

The present article is organized as follows. In Section 2, we review some mathematical background material on the algebra of representation functions of a compact Lie group, we construct the spherical functions of $S^{3}$, and we introduce our notation for abstract 2complexes. The Barrett-Crane model and $S O(4)$ lattice $B F$-theory are introduced in Section 3. In Section 4, we present the duality transformation for the Barrett-Crane model in detail and show how this model is related to $S O(4)$ lattice $B F$-theory subject to a suitable constraint. We also discuss its geometric interpretation and the possible choices of face and edge amplitudes. Section 5 finally contains our concluding comments.

Note added: The Barrett-Crane model is often understood as a path integral in the 'real time' picture, i.e. using an integrand of the form $\exp (i S)$ for some action $S$. However, Baez and Christensen [22] have shown that for the common choices of edge amplitudes, the integrand of the partition function is always positive so that the path integral admits an interpretation in the framework of Statistical Mechanics in terms of probabilities. In this article, we use the language of Statistical Mechanics.

\section{Preliminaries}

In this section, we briefly summarize definitions and some basic statements related to the algebra of representation functions $C_{\text {alg }}(G)$ of $G$ where $G$ is a compact Lie group. Furthermore, we describe the spherical functions of $S^{3}$ and present our notation for the $S O(4)$ and $S U(2) \times S U(2)$ actions on $S^{3}$. More background material and most of the proofs can be found, for example, in 23, 24. Finally, we introduce the 2-complexes that we employ in our definition of the Barrett-Crane model and in the duality transformation.

\subsection{The Hopf algebra of representation functions}

Let $G$ be a compact Lie group. We denote finite-dimensional complex vector spaces on which $G$ is represented by $V_{\rho}$ and by $\rho: G \rightarrow$ Aut $V_{\rho}$ the corresponding group homomorphisms. Since each finite-dimensional complex representation of $G$ is equivalent to a unitary representation, we select a set $\widetilde{\mathcal{R}}$ containing one unitary representation of $G$ for each equivalence class of finite-dimensional representations. The tensor product, the direct sum and taking the dual are supposed to be closed operations on this set. This amounts to a particular choice of representation isomorphisms $\rho_{1} \otimes \rho_{2} \leftrightarrow \rho_{3}$ etc., $\rho_{j} \in \widetilde{\mathcal{R}}$, which is implicit in our formulas. We furthermore denote by $\mathcal{R} \subseteq \widetilde{\mathcal{R}}$ the subset of irreducible representations. 
For a representation $\rho \in \widetilde{\mathcal{R}}$, the dual representation is denoted by $\rho^{*}$, and the dual vector space of $V_{\rho}$ by $V_{\rho}^{*}$. The dual representation is given by $\rho^{*}: G \mapsto$ Aut $V_{\rho}^{*}$, where

$$
\rho^{*}(g): V_{\rho}^{*} \rightarrow V_{\rho}^{*}, \quad \eta \mapsto \eta \circ \rho\left(g^{-1}\right),
$$

i.e. $\left(\rho^{*}(g) \eta\right)(v)=\eta\left(\rho\left(g^{-1}\right) v\right)$ for all $v \in V_{\rho}$. There exists a one-dimensional 'trivial' representation of $G$ which is denoted by $V_{[1]} \cong \mathbb{C}$.

For the unitary representations $V_{\rho}, \rho \in \widetilde{\mathcal{R}}$, we have standard (sesquilinear) scalar products $\langle\cdot ; \cdot\rangle$ and orthonormal bases $\left\{v_{j}\right\}_{j}$. For each basis $\left\{v_{j}\right\}_{j}$ of $V_{\rho}$, there exists a dual basis $\left\{\eta^{j}\right\}_{j}$ of $V_{\rho}^{*}$ so that $\eta^{j}\left(v_{k}\right)=\left\langle v_{j} ; v_{k}\right\rangle$, and $V_{\rho}^{*}$ possesses a scalar product such that $\left\langle\eta^{j} ; \eta^{k}\right\rangle=\eta^{k}\left(v_{j}\right)$, $1 \leq j, k \leq \operatorname{dim} V_{\rho}$.

The complex-valued functions

$$
t_{\eta, v}^{(\rho)}: G \rightarrow \mathbb{C}, \quad g \mapsto \eta(\rho(g) v),
$$

where $\rho \in \widetilde{\mathcal{R}}, v \in V_{\rho}$ and $\eta \in V_{\rho}^{*}$, are called the representation functions of $G$. They form a commutative and associative unital algebra over $\mathbb{C}$,

$$
C_{\text {alg }}(G):=\left\{t_{\eta, v}^{(\rho)}: \quad \rho \in \widetilde{\mathcal{R}}, v \in V_{\rho}, \eta \in V_{\rho}^{*}\right\},
$$

whose operations are given by

$$
\begin{aligned}
\left(t_{\eta, v}^{(\rho)}+t_{\vartheta, w}^{(\sigma)}\right)(g) & :=t_{\eta+\vartheta, v+w}^{(\rho \oplus \sigma)}(g), \\
\left(t_{\eta, v}^{(\rho)} \cdot t_{\vartheta, w}^{(\sigma)}\right)(g) & :=t_{\eta \otimes \vartheta, v \otimes w}^{(\rho \otimes \sigma)}(g),
\end{aligned}
$$

where $\rho, \sigma \in \widetilde{\mathcal{R}}, v \in V_{\rho}, w \in V_{\sigma}, \eta \in V_{\rho}^{*}, \vartheta \in V_{\sigma}^{*}$ and $g \in G$. The zero element of $C_{\text {alg }}(G)$ is given by $t_{0,0}^{[1]}(g)=0$ and its unit element by $t_{\eta, v}^{[1]}(g)=1$.

The algebra $C_{\text {alg }}(G)$ is furthermore equipped with a Hopf algebra structure employing the coproduct $\Delta: C_{\text {alg }}(G) \rightarrow C_{\text {alg }}(G) \otimes C_{\text {alg }}(G) \cong C_{\text {alg }}(G \times G)$, the co-unit $\varepsilon: C_{\text {alg }}(G) \rightarrow \mathbb{C}$ and the antipode $S: C_{\text {alg }}(G) \rightarrow C_{\text {alg }}(G)$ which are defined by

$$
\begin{aligned}
\Delta t_{\eta, v}^{(\rho)}(g, h) & :=t_{\eta, v}^{(\rho)}(g \cdot h), \\
\varepsilon t_{\eta, v}^{(\rho)} & :=t_{\eta, v}^{(\rho)}(e) \\
S t_{\eta, v}^{(\rho)}(g) & :=t_{\eta, v}^{(\rho)}\left(g^{-1}\right)
\end{aligned}
$$

for $\rho \in \widetilde{\mathcal{R}}, v \in V_{\rho}, \eta \in V_{\rho}^{*}$ and $g, h \in G$. Here $e \in G$ denotes the group unit.

In the standard orthonormal bases, the representation functions are given by the coefficients of representation matrices,

$$
t_{m n}^{(\rho)}(g)=\eta^{m}\left(\rho(g) v_{n}\right)=\left\langle v_{m} ; \rho(g) v_{n}\right\rangle=\rho(g)_{m n},
$$

so that the coproduct corresponds to the matrix product,

$$
\Delta t_{m n}^{(\rho)}(g, h)=\sum_{j=1}^{\operatorname{dim} V_{\rho}} t_{m j}^{(\rho)}(g) t_{j n}^{(\rho)}(h)=\sum_{j=1}^{\operatorname{dim} V_{\rho}} \rho(g)_{m j} \rho(h)_{j n},
$$


while the antipode refers to the inverse matrix, $S t_{m n}^{(\rho)}(g)=\left(\rho(g)^{-1}\right)_{m n}$, and the co-unit describes the coefficients of the unit matrix, $\varepsilon t_{m n}^{(\rho)}=\delta_{m n}$. Furthermore, the antipode relates a representation to its dual,

$$
S t_{m n}^{(\rho)}(g)=\eta^{m}\left(\rho(g)^{-1} v_{n}\right)=\left(\rho^{*}(g) \eta^{m}\right)\left(v_{n}\right)=\left\langle\eta^{n} ; \rho^{*}(g) \eta^{m}\right\rangle=t_{n m}^{\left(\rho^{*}\right)}(g),
$$

which, in the case of unitary $\rho$, is just the conjugate representation because on the other hand $S t_{m n}^{(\rho)}(g)=\overline{\rho(g)_{n m}}=\overline{t_{n m}^{(\rho)}(g)}$. Here the bar denotes complex conjugation.

\subsection{Peter-Weyl decomposition and theorem}

The structure of the algebra $C_{\mathrm{alg}}(G)$ can be understood if $C_{\mathrm{alg}}(G)$ is considered as a representation of $G \times G$ by combined left and right translation of the function argument,

$$
(G \times G) \times C_{\text {alg }}(G) \rightarrow C_{\text {alg }}(G), \quad\left(\left(g_{1}, g_{2}\right), f\right) \mapsto\left(h \mapsto f\left(g_{1}^{-1} h g_{2}\right)\right) .
$$

It can then be decomposed into its irreducible components as a representation of $G \times G$.

Theorem 2.1 (Peter-Weyl decomposition). Let $G$ be a compact Lie group.

1. There is an isomorphism

$$
C_{\mathrm{alg}}(G) \cong \bigoplus_{\rho \in \mathcal{R}}\left(V_{\rho} \otimes V_{\rho}^{*}\right)
$$

of representations of $G \times G$. Here the direct sum runs over the equivalence classes of finite-dimensional irreducible representations of $G$. The direct summands $V_{\rho} \otimes V_{\rho}^{*}$ are irreducible as representations of $G \times G$.

2. The direct sum in (2.10) is orthogonal with respect to the $L^{2}$-scalar product on $C_{\text {alg }}(G)$ which is formed using the Haar measure of $G$ on the left hand side, and using the standard scalar products on the right hand side, namely

$$
\left\langle t_{\eta, v}^{(\rho)} ; t_{\vartheta, w}^{(\sigma)}\right\rangle_{L^{2}}:=\int_{G} \overline{t_{\eta, v}^{(\rho)}(g)} \cdot t_{\vartheta, w}^{(\sigma)}(g) d g=\frac{1}{\operatorname{dim} V_{\rho}} \delta_{\rho \sigma} \overline{\langle\eta ; \vartheta\rangle}\langle v ; w\rangle,
$$

where $\rho, \sigma \in \mathcal{R}$ are irreducible. The Haar measure is denoted by $\int_{G} d g$ and normalized so that $\int_{G} d g=1$.

Corollary 2.2. Each representation function $f \in C_{\text {alg }}(G)$ can be decomposed according to (2.10),

$$
f(g)=\sum_{\rho \in \mathcal{R}} \sum_{m, n=1}^{\operatorname{dim} V_{\rho}} \widehat{f}_{m n}^{(\rho)} t_{m n}^{(\rho)}(g), \quad \text { where } \quad \widehat{f}_{m n}^{(\rho)}=\operatorname{dim} V_{\rho} \int_{G} \overline{t_{m n}^{(\rho)}(g)} f(g) d g .
$$

The analytical aspects of $C_{\mathrm{alg}}(G)$ can now be stated.

Theorem 2.3 (Peter-Weyl Theorem). Let $G$ be a compact Lie group. Then $C_{\text {alg }}(G)$ is dense in $L^{2}(G)$ with respect to the $L^{2}$ norm.

We use the Peter-Weyl theorem in order to complete $C_{\text {alg }}(G)$ with respect to the $L^{2}$ norm to $L^{2}(G)$. Functions $f \in L^{2}(G)$ then correspond to square summable series in (2.12). These series are therefore invariant under a reordering of summands, and their limits commute with group integrations. We make use of these invariances in the duality transformation. 


\subsection{Character decomposition}

The characters of $G$ are the algebraic class functions, i.e. those functions $f \in C_{\text {alg }}(G)$ that satisfy $f\left(h g h^{-1}\right)=f(g)$ for all $g, h \in G$.

Proposition 2.4. For class functions $f \in C_{\text {alg }}(G)$, the Peter-Weyl decomposition (2.12) specializes to the character decomposition

$$
f(g)=\sum_{\rho \in \mathcal{R}} \chi^{(\rho)}(g) \widehat{f}_{\rho}, \quad \text { where } \quad \widehat{f}_{\rho}=\int_{G} \overline{\chi^{(\rho)}(g)} f(g) d g .
$$

Here

$$
\chi^{(\rho)}:=\sum_{n=1}^{\operatorname{dim} V_{\rho}} t_{n n}^{(\rho)}
$$

denotes the character of the representation $\rho \in \widetilde{\mathcal{R}}$. For irreducible $\rho, \sigma \in \mathcal{R}$, the orthogonality relation (2.11) implies,

$$
\left\langle\chi^{(\rho)} ; \chi^{(\sigma)}\right\rangle_{L^{2}}=\int_{G} \overline{\chi^{(\rho)}(g)} \chi^{(\sigma)}(g) d g=\delta_{\rho \sigma}
$$

The formal $\delta$-element,

$$
\delta(g):=\sum_{\rho \in \mathcal{R}} \operatorname{dim} V_{\rho} \cdot \chi^{(\rho)}(g)
$$

satisfies for all $f \in C_{\text {alg }}(G)$,

$$
\int_{G} f(g) \delta\left(g h^{-1}\right) d g=f(h)
$$

\subsection{Diagrams}

In order to present the partition function of lattice gauge theory both in the original formulation using group integrations and in the dual formulation [8, 10] as a spin foam model, we define a particular intertwiner [11] based on the Haar measure.

Definition 2.5. Let $G$ be a compact Lie group and $\rho_{1}, \ldots, \rho_{r} \in \mathcal{R}, r \in \mathbb{N}$, be finitedimensional irreducible representations of $G$. The Haar intertwiner,

$$
T: \bigotimes_{\ell=1}^{r} V_{\rho_{\ell}} \rightarrow \bigotimes_{\ell=1}^{r} V_{\rho_{\ell}}
$$

is defined by its matrix elements,

$$
T_{m_{1} m_{2} \ldots m_{r} ; n_{1} n_{2} \ldots n_{r}}:=\int_{G} t_{m_{1} n_{1}}^{\left(\rho_{1}\right)}(g) t_{m_{2} n_{2}}^{\left(\rho_{2}\right)}(g) \cdots t_{m_{r} n_{r}}^{\left(\rho_{r}\right)}(g) d g .
$$




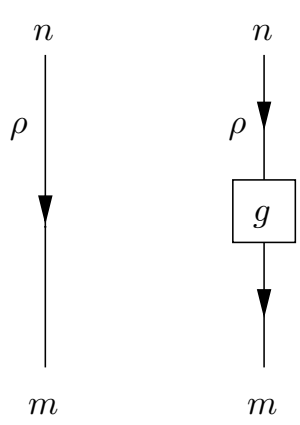

(a)

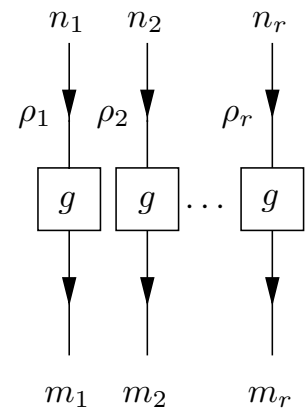

(c)

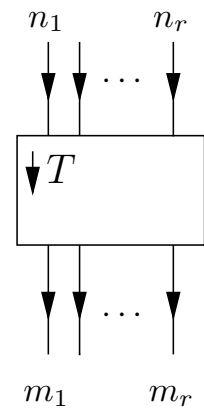

(d)

Figure 1: Diagrams to visualize the index structure in the calculation of group integrals. (a) The identity map of $V_{\rho}$; (b) a representation function $t_{m n}^{(\rho)}$; (c) a product of representation functions $t_{m_{1} n_{1}}^{\left(\rho_{1}\right)} \cdots t_{m_{r} n_{r}}^{\left(\rho_{r}\right)}$; (d) the Haar intertwiner.

Lemma 2.6. The Haar intertwiner $T$ of (2.18) satisfies for all $h \in G$,

$$
\begin{aligned}
T & =\int_{G}\left(\rho_{1}(g) \otimes \cdots \otimes \rho_{r}(g)\right) d g \\
T & =\left(\rho_{1}(h) \otimes \cdots \otimes \rho_{r}(h)\right) \circ T=T \circ\left(\rho_{1}(h) \otimes \cdots \otimes \rho_{r}(h)\right), \\
T \circ T & =T
\end{aligned}
$$

The first equation is just the definition while the second and third are consequences of the left-right translation invariance of the Haar measure. In particular, $T$ forms a morphism of representations of $G$.

In the subsequent sections, the Haar intertwiner will appear in rather complicated calculations. It is therefore convenient to introduce diagrams that visualize the structure of the indices in these formulas (Figure 1).

The diagrams are read from top to bottom. We draw directed lines which are labelled with irreducible representations $\rho \in \mathcal{R}$ of $G$. If the arrow points down, the line denotes the identity map of $V_{\rho}$, Figure 1(a). If the arrow points up, it refers to the identity map of the dual representation $V_{\rho}^{*}$. A representation function $t_{m n}^{(\rho)}$ is denoted by a box with an incoming and an outgoing line (b), and a product of representation functions by boxes placed next to each other (c). The Haar intertwiner is visualized by the box labelled $T$ in (d).

\subsection{Spherical functions of $S^{3}$}

We construct the spherical functions of $S^{3}$ from the identification $S^{3} \cong S U(2)$, i.e. we can make use of the representations functions of $S U(2)$. The finite-dimensional irreducible representations of $S U(2)$ are characterized by half-integers $j \in \frac{1}{2} \mathbb{N}_{0}$. We write $V_{j}, \operatorname{dim} V_{j}=2 j+1$, for the irreducible representations. Using the Peter-Weyl decomposition (2.12), we can give 
a basis for $C_{\mathrm{alg}}(S U(2))$,

$$
\left\{t_{m n}^{(j)}: S U(2) \rightarrow \mathbb{C}: \quad j \in \frac{1}{2} \mathbb{N}_{0}, \quad 1 \leq m, n \leq 2 j+1\right\} .
$$

In particular, we obtain the (algebraic) spherical functions of $S^{3}$ from the identification $S^{3} \cong S U(2)$ as

$$
H_{m n}^{(j)}: S^{3} \rightarrow \mathbb{C}, g \mapsto t_{m n}^{(j)}(g),
$$

for $j \in \frac{1}{2} \mathbb{N}_{0}$ and $1 \leq m, n \leq 2 j+1$.

We also wish to describe the transitive actions of $S O(4)$ and $\operatorname{Spin}(4) \cong S U(2) \times S U(2)$ on $S^{3}$ for which the stabilizer of a point $g \in S^{3}$ is $S O(3)$ or $S U(2)$, respectively. Writing $2 \times 2$ complex matrices with the usual properties for the elements of $S U(2)$, we obtain $S O(3)$ as the quotient $S O(3):=S U(2) / \mathbb{Z}_{2}$ where $\mathbb{Z}_{2}:=\{-\mathbb{1}, \mathbb{1}\} \leq S U(2)$ and $S O(4):=(S U(2) \times$ $S U(2)) / \mathbb{Z}_{2}$ where $\mathbb{Z}_{2}:=\{(\mathbb{1}, \mathbb{1}),(-\mathbb{1},-\mathbb{1})\} \leq S U(2) \times S U(2)$. The relevant actions are the following.

Proposition 2.7. The map

$$
(S U(2) \times S U(2)) \times S^{3} \rightarrow S^{3},\left(\left(g_{1}, g_{2}\right), g\right) \mapsto\left(g_{1}, g_{2}\right) * g:=g_{1} g g_{2}^{-1},
$$

is a transitive group action. The stabilizer of a point $g \in S^{3}$ is given by

$$
\operatorname{Stab}_{S U(2) \times S U(2)}(g):=\left\{\left(g h g^{-1}, h\right): \quad h \in S U(2)\right\},
$$

and forms a subgroup of $S U(2) \times S U(2)$ via the inclusion

$$
\imath_{g}^{\prime}: S U(2) \hookrightarrow S U(2) \times S U(2), h \mapsto\left(g h g^{-1}, h\right) .
$$

The action $*$ on $S^{3}$ is well defined for the quotient $S O(4) \cong(S U(2) \times S U(2)) / \mathbb{Z}_{2}$ in which case the stabilizer is isomorphic to $S O(3)$ and given by the inclusion

$$
\imath_{g}: S O(3) \hookrightarrow S O(4), h \mapsto\left(g h g^{-1}, h\right),
$$

which we have written down for representatives.

Corollary 2.8. The algebra of spherical functions $C_{\text {alg }}\left(S^{3}\right)$ forms a representation of $S U(2) \times$ $S U(2)$ by translation of the function argument,

$$
\left.(S U(2) \times S U(2)) \times C_{\mathrm{alg}}\left(S^{3}\right) \rightarrow C_{\mathrm{alg}}\left(S^{3}\right),\left(\left(g_{1}, g_{2}\right), f\right) \mapsto\left(g \mapsto f\left(\left(g_{1}, g_{2}\right) * g\right)=f\left(g_{1} g g_{2}^{-1}\right)\right)\right),
$$

which also gives rise to a representation of $S O(4) \cong(S U(2) \times S U(2)) / \mathbb{Z}_{2}$.

The decomposition of $C_{\text {alg }}\left(S^{3}\right)$ into irreducible representations of $S U(2) \times S U(2)$ coincides with the Peter-Weyl decomposition (2.10) of $S U(2)$,

$$
C_{\mathrm{alg}}\left(S^{3}\right) \cong \cong_{S U(2) \times S U(2)} \bigoplus_{j \in \frac{1}{2} \mathbb{N}_{0}}\left(V_{j} \otimes V_{j}^{*}\right),
$$

where the direct summand $V_{j} \otimes V_{j}^{*}$ for a given $j$ has the basis

$$
\left\{H_{m_{1} m_{2}}^{(j)}: \quad 1 \leq m_{1}, m_{2} \leq 2 j+1\right\} .
$$

In our notation, the spherical functions $H_{m n}^{(j)}$ therefore transform as the coefficients of a vector in $V_{j} \otimes V_{j}^{*} \cong \operatorname{Hom}_{S U(2)}\left(V_{j}, V_{j}\right)$. 
Furthermore, the Haar measure of $S U(2)$ provides an integral over $S^{3}$ with the desired translation invariance under the $S O(4)$-action.

Proposition 2.9. Let $f \in C_{\mathrm{alg}}\left(S^{3}\right)$ and $\left(g_{1}, g_{2}\right) \in S U(2) \times S U(2)$. Then the integral over $S^{3}$, obtained from the Haar measure of $S U(2)$ under the identification $S^{3} \cong S U(2)$, satisfies

$$
\int_{S^{3}} f(g) d g=\int_{S^{3}} f\left(\left(g_{1}, g_{2}\right) * g\right) d g .
$$

There is a scalar product on $C_{\text {alg }}\left(S^{3}\right)$,

$$
\left\langle f_{1}, f_{2}\right\rangle:=\int_{S^{3}} \overline{f_{1}(g)} f_{2}(g) d g,
$$

with respect to which the spherical functions are orthogonal,

$$
\left\langle H_{m n}^{(j)}, H_{p q}^{(k)}\right\rangle=\frac{1}{2 j+1} \delta_{j k} \delta_{m p} \delta_{n q}
$$

where $j, k \in \frac{1}{2} \mathbb{N}_{0}$ and $1 \leq m, n \leq 2 j+1,1 \leq p, q \leq 2 k+1$.

We also construct the algebras of functions on $S U(2) \times S U(2)$ and $S O(4)$. We denote the irreducible representations of $S U(2) \times S U(2)$ by pairs $\left(j_{1}, j_{2}\right)$ of half-integers $j_{1}, j_{2} \in \frac{1}{2} \mathbb{N}_{0}$. They are of the form $V_{\left(j_{1}, j_{2}\right)}=V_{j_{1}} \otimes V_{j_{2}}$. A basis for the algebra $C_{\text {alg }}(S U(2) \times S U(2))$ is given by the functions

$$
t_{m_{1} m_{2}, n_{1} n_{2}}^{\left(j_{1}, j_{2}\right)}: S U(2) \times S U(2) \rightarrow \mathbb{C},\left(g_{1}, g_{2}\right) \mapsto t_{m_{1} n_{1}}^{\left(j_{1}\right)}\left(g_{1}\right) \cdot t_{m_{2} n_{2}}^{\left(j_{2}\right)}\left(g_{2}\right),
$$

where $j_{1}, j_{2} \in \frac{1}{2} \mathbb{N}_{0}, 1 \leq m_{\ell}, n_{\ell} \leq 2 j_{\ell}+1$. It is known that the functions $t_{m_{1} m_{2}, n_{1} n_{2}}^{\left(j_{1}, j_{2}\right)}$ are well defined on $S O(4) \cong(S U(2) \times S U(2)) / \mathbb{Z}_{2}$ if and only if $j_{1}+j_{2} \in \mathbb{N}_{0}$, i.e. if in $V_{\left(j_{1}, j_{2}\right)}=V_{j_{1}} \otimes V_{j_{2}}$ either both factors are even- or both are odd-dimensional. Therefore $t_{m_{1} m_{2}, n_{1} n_{2}}^{\left(j_{1}, j_{2}\right)} \in C_{\text {alg }}(S O(4))$ if and only if $j_{1}+j_{2} \in \mathbb{N}_{0}$.

Usually, the spherical functions of $S^{3}$ are constructed as the functions on $S O(4) / S O(3)$, i.e. as the functions on $S O(4)$ that are constant on cosets of $S O(3)$ [23]. There exist representation functions $t_{m n, p q}^{\left(j_{1}, j_{2}\right)} \in C_{\text {alg }}(S O(4))$ that are constant on these cosets only if $j_{1}=j_{2}$, i.e. in the class-1 representations of $S O(4)$ with respect to $S O(3)$. These are the balanced representations $V_{(j, j)}=V_{j} \otimes V_{j}$.

Our spherical functions (2.23), however, transform as the coefficients of vectors in $V_{\left(j, j^{*}\right)}=$ $V_{j} \otimes V_{j}^{*}$. Recall that for $S U(2)$, there exist isomorphisms of representations $V_{j} \cong V_{j}^{*}$ (which we have not explicitly constructed) which can be applied to the right tensor factor in order to relate our spherical functions to the standard construction.

\subsection{Two-complexes}

In order to define lattice gauge theory and the Barrett-Crane model, we need essentially an abstract oriented 2-complex. This is a collection of faces, edges and vertices together with a boundary relation specifying which edges are contained in the boundary of a given face, etc.. For the partition function of lattice gauge theory, we require a formulation which explicitly describes the cyclic ordering of the edges in the boundary of a given face. This is achieved by the following definition of a 2-complex with cyclic structure. 

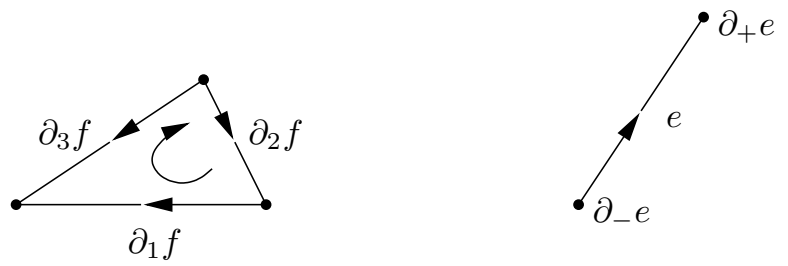

Figure 2: The maps $\partial_{ \pm}, \partial_{j}$ and $\varepsilon_{j}$ and the conditions (2.36). Here $N(f)=3$, $\varepsilon_{1} f=+1, \varepsilon_{2} f=+1$ and $\varepsilon_{3} f=(-1)$.

Definition 2.10. A finite 2-complex with cyclic structure $(V, E, F)$ consists of finite sets $V$ (vertices), $E$ (edges) and $F$ (faces) together with maps

$$
\begin{aligned}
& \partial_{+}: E \rightarrow V, \quad \text { (end point of an edge) } \\
& \partial_{-}: E \rightarrow V, \quad \text { (starting point of an edge) } \\
& N: F \rightarrow \mathbb{N}, \quad \text { (number of edges in the boundary of a face) } \\
& \partial_{j}: F \rightarrow E, \quad \text { (the } j \text {-th edge in the boundary of a face) } \\
& \varepsilon_{j}: F \rightarrow\{-1,+1\}, \quad \text { (its orientation) }
\end{aligned}
$$

so that

$$
\begin{gathered}
\partial_{-\varepsilon_{j} f} \partial_{j} f=\partial_{\varepsilon_{j+1} f} \partial_{j+1} f, \quad 1 \leq j \leq N(f)-1, \\
\partial_{-\varepsilon_{N(f)} f} \partial_{N(f)} f=\partial_{\varepsilon_{1} f} \partial_{1} f
\end{gathered}
$$

for all $f \in F$.

Remark 2.11. 1. The conditions (2.36) state that the edges in the boundary of a face $f \in F$ are in cyclic ordering from $\partial_{N(f)} f$ to $\partial_{1} f$ where one encounters the edges with a relative orientation given by $\varepsilon_{j} f$, see Figure 2. Observe that (2.36) contains combinatorial information similar to the condition $\partial \circ \partial=0$ on the boundary operator $\partial$ in Abelian simplicial homology.

2. For the existence of the partition functions in the following sections, we require that the 2-complexes have only a finite number of vertices, edges and faces.

In the subsequent calculations, it is convenient to use the following abbreviations.

Definition 2.12. Let $(V, E, F)$ denote a finite combinatorial 2-complex with cyclic structure. For a given edge $e \in E$, the sets

$$
\begin{aligned}
& e_{+}:=\left\{f \in F: \quad e=\partial_{j} f, \quad \varepsilon_{j} f=(+1) \quad \text { for some } j, \quad 1 \leq j \leq N(f)\right\}, \\
& e_{-}:=\left\{f \in F: \quad e=\partial_{j} f, \quad \varepsilon_{j} f=(-1) \quad \text { for some } j, \quad 1 \leq j \leq N(f)\right\} \text {, }
\end{aligned}
$$

contain all faces that have the edge $e$ in their boundary with positive $(+)$ or negative $(-)$ orientation, and we write $\delta e:=e_{+} \cup e_{-}$. For a given face $f \in F$, the set

$$
f_{0}:=\left\{v \in V: \quad v=\partial_{-} \partial_{j} f \quad \text { for some } j, \quad 1 \leq j \leq N(f)\right\},
$$


denotes all vertices that belong to the boundary of the face $f$. Finally, the sets

$$
\begin{aligned}
& f_{+}:=\left\{e \in E: \quad e=\partial_{j} f, \quad \varepsilon_{j} f=(+1) \quad \text { for some } j, \quad 1 \leq j \leq N(f)\right\}, \\
& f_{-}:=\left\{e \in E: \quad e=\partial_{j} f, \quad \varepsilon_{j} f=(-1) \quad \text { for some } j, \quad 1 \leq j \leq N(f)\right\},
\end{aligned}
$$

contain all edges in the boundary of the face $f$ that occur with positive $(+)$ or negative $(-)$ orientation, and $\partial f:=f_{+} \cup f_{-}$. Finally, for a given face $f \in F, v_{+}(f)$ denotes the edge $e \in \partial f$ for which $v=\partial_{+} e$, and similarly, $v_{-}(f)$ the edge $e \in \partial f$ for which $v=\partial_{-} e$.

Remark 2.13. Given any triangulation of a four-manifold, we can construct a 2-complex with cyclic structure that is combinatorially dual to the triangulation. The vertices $V$ are the four-simplices of the triangulation, the edges $E$ the tetrahedra and the faces $F$ the triangles. An edge is contained in the boundary of a given face whenever the corresponding tetrahedron contains the triangle in its boundary, etc.. A cyclic structure can then be constructed by induction. In particular, if the triangulation contains only finitely many four-simplices, we obtain a finite 2-complex with cyclic structure.

\section{The Barrett-Crane model}

When we present the definition of the Barrett-Crane model [4, 可], we wish to emphasize its structural similarity to lattice gauge theory with pure gauge fields, in particular to $S O(4)$ lattice $B F$-theory. Therefore we recall the two formulations of lattice gauge theory, the original one using group integrations and the dual formulation 8, 10] as a spin foam model before we present the definition of the class of Barrett-Crane models which we consider in this article.

\subsection{Lattice gauge theory}

For more background on lattice gauge theory, we refer the reader to text books such as [25,26]. The partition function of lattice gauge theory can be formulated on any 2-complex with cyclic structure as follows.

Definition 3.1. Let $G$ be a compact Lie group and $(V, E, F)$ be a finite 2-complex with cyclic structure. The partition function of lattice gauge theory is defined as

$$
Z=\left(\prod_{e \in E} \int_{G} d g_{e}\right) \prod_{f \in F} w\left(g_{f}\right), \quad g_{f}:=g_{\partial_{1} f}^{\varepsilon_{1} f} \cdots g_{\partial_{N(f)} f}^{\varepsilon_{N(f)} f} .
$$

Here $w: G \rightarrow \mathbb{R}$ is the local Boltzmann weight which is required to be a positive and $L^{2}$ integrable class function of $G$ that satisfies $w\left(g^{-1}\right)=w(g)$.

Remark 3.2. 1. Our notation in (3.1) means that there is one integral $\int_{G} d g$ for each edge $e \in E$. We use this notation very often for integrals and sums in the subsequent calculations.

2. The partition function of lattice gauge theory is an integral over the group $G$ for each edge of the 2-complex so that the set of configurations is $G^{E}$. The Boltzmann weight is calculated for each face where $g_{f}$ denotes the ordered product of group elements assigned to the edges in the boundary of the face. This is the place where the cyclic structure of the 2-complex enters the definition. 


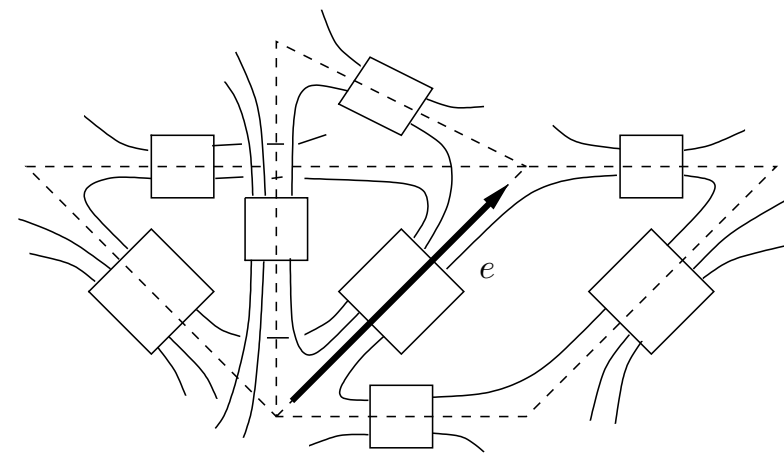

(a)

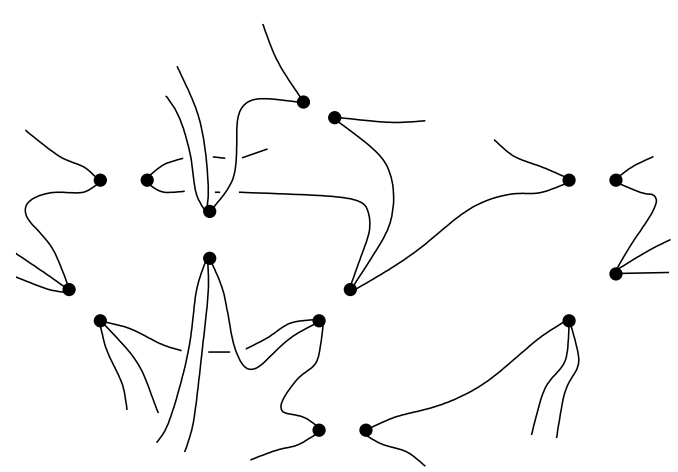

(b)

Figure 3: An edge $e \in E$ in the boundary of three faces, two triangles and one quadrilateral. (a) Equation (3.3) contains one Haar intertwiner for each edge. The intertwiners are represented by the boxes as in Figure 1 1 (d), and the diagram indicates how to contract the indices. (b) The contraction of indices in the BarrettCrane model (3.10). The full dots represent the Barrett-Crane intertwiners (3.6). We have omitted all arrows in both diagrams (a) and (b).

3. The value of the partition function is independent of the choice of cyclic structure. The starting point for the cyclic numbering of edges in the boundary of a face does not matter because the Boltzmann weight is given by a class function and thus invariant under cyclic permutation of the factors of $g_{f}$. Reversal of the orientation is also a symmetry because it replaces $g_{f}$ by $g_{f}^{-1}$.

4. For lattice Yang-Mills theory, the Boltzmann weight is given by $w(g)=\exp (-s(g))$ in terms of the Euclidean action $s: G \rightarrow \mathbb{R}$. For lattice $B F$-theory [21], however, we have $w(g)=\delta(g)$. For a generic Lie group $G$ and a generic 2-complex, the partition function is therefore not well defined.

The Boltzmann weight of lattice gauge theory is invariant under local gauge transformations.

Lemma 3.3. Let $h: V \rightarrow G, v \mapsto h_{v}$ associate a group element to each vertex. The Boltzmann weight $w\left(g_{f}\right)$ is invariant under the local gauge transformations,

$$
G^{E} \rightarrow G^{E}, g_{e} \mapsto h_{\partial_{+} e} \cdot g_{e} \cdot h_{\partial_{-} e}^{-1}
$$

Proof. In order to prove this invariance, one has to make use of the conditions (2.36) and of the fact that $w$ is a class function.

There exists a dual formulation of lattice gauge theory in which the partition function is no longer given by group integrals but rather by sums over representations and intertwiners 8 , 10,11, so that it forms a spin foam model. We present a definition of the dual model which coincides with an intermediate step in [8, 10] and directly corresponds to the diagrammatical formulation of 11. 
Theorem 3.4. Let $G$ be a compact Lie group and $(V, E, F)$ be a finite 2 -complex with cyclic structure. The partition function of lattice gauge theory (3.1) is equal to

$$
\begin{aligned}
Z= & \left(\prod_{f \in F} \sum_{\rho_{f} \in \mathcal{R}}\right)\left(\prod_{f \in F} \widehat{w}_{\rho_{f}}\right)\left(\prod_{f \in F} \prod_{v \in f_{0}} \sum_{n(f, v)=1}^{\operatorname{dim} V_{\rho_{f}}}\right) \\
& \times \prod_{e \in E} \underbrace{T_{n(f)}^{(e)}}_{f \in e_{+}} \underbrace{n\left(f, \partial_{+} e\right) \ldots}_{f \in e_{-}} \underbrace{n\left(f, \partial_{+} e\right) \ldots}_{f \in e_{+}} \underbrace{n\left(f, \partial_{-} e\right) \ldots}_{f \in e_{-}} \underbrace{n\left(f, \partial_{-} e\right) \ldots}
\end{aligned}
$$

where $T^{(e)}$ denotes the Haar intertwiner 2.18) for the following representations,

$$
T^{(e)}:\left(\bigotimes_{f \in e_{+}} V_{\rho_{f}}\right) \otimes\left(\bigotimes_{f \in e_{-}} V_{\rho_{f}}^{*}\right) \rightarrow\left(\bigotimes_{f \in e_{+}} V_{\rho_{f}}\right) \otimes\left(\bigotimes_{f \in e_{-}} V_{\rho_{f}}^{*}\right) .
$$

We require here that the ordering of tensor factors parameterized by $f \in e_{ \pm}$agrees with the ordering of indices in (3.3). The coefficients $\widehat{w}_{\rho}$ are the coefficients of the character expansion of the Boltzmann weight,

$$
\widehat{w}_{\rho}=\int_{G} \overline{\chi^{(\rho)}(g)} w(g) d g .
$$

Remark 3.5. 1. The dual form of the partition function is a sum over all colourings of faces $f \in F$ with irreducible representations $\rho \in \mathcal{R}$ of $G$. There is a Haar intertwiner $T^{(e)}$ assigned to each edge $e \in E$, and under the sum we have a weight $\widehat{w}_{\rho}$ for each face. The sums over the indices $n(f, v)$ for each face $f \in F$ and each vertex $v \in f_{0}$ in the boundary of the face, contract the indices of the Haar intertwiners. This is illustrated in Figure 3(a).

2. For lattice $B F$-theory, we have $\widehat{w}_{\rho}=\operatorname{dim} V_{\rho}$, i.e. the sums do not converge. In particular, for $G=S U(2)$ and a 2-complex that is dual to a given triangulation of a four-manifold, we obtain the Ooguri model [20] whose $S O(4)$ version we will consider later.

3. For lattice Yang-Mills theory, the transformation relating (3.1) to (3.3) is a strong-weak or high temperature-low temperature duality transformation [8, 9 . This is a consequence of the fact that the character expansion of the Boltzmann weight has many similarities to Fourier decomposition and maps a Boltzmann weight with a wide peak to a function with a narrow peak and conversely.

\subsection{The Barrett-Crane intertwiner}

The Barrett-Crane model has close similarities to the spin foam model dual to $S O(4)$ lattice $B F$-theory. We obtain the Barrett-Crane model starting from the partition function (3.3) if we restrict the sum over irreducible representations to the balanced representations of $S O(4)$. Furthermore, we have to replace the Haar intertwiner of (3.3) by a pair of Barrett-Crane intertwiners.

Following Barrett, Freidel and Krasnov 18, 19], the Barrett-Crane intertwiner can be written as an integral over $S^{3}$. 
Definition 3.6. Let $V_{\left(j_{\ell}, j_{\ell}^{*}\right)}$ and $V_{\left(k_{\ell}, k_{\ell}^{*}\right)}, j_{\ell}, k_{\ell} \in \frac{1}{2} \mathbb{N}_{0}$, denote balanced irreducible representations of $S O(4)$ whose right tensor factor has been dualized. The Barrett-Crane intertwiner

$$
I: \bigotimes_{\ell=1}^{r} V_{\left(j_{\ell}, j_{\ell}^{*}\right)} \rightarrow \bigotimes_{\ell=1}^{s} V_{\left(k_{\ell}, k_{\ell}^{*}\right)}
$$

is defined in terms of the matrix elements,

$$
I_{\left(m_{1} n_{1}\right) \ldots ;\left(p_{1} q_{1}\right) \ldots}:=\int_{S^{3}} \overline{H_{p_{1} q_{1}}^{\left(j_{1}\right)}(x) \cdots H_{p_{r} q_{r}}^{\left(j_{r}\right)}(x)} H_{m_{1} n_{1}}^{\left(k_{1}\right)}(x) \cdots H_{m_{s} n_{s}}^{\left(k_{s}\right)}(x) d x,
$$

of the intertwiner

$$
\mathbb{C} \rightarrow\left(\bigotimes_{\ell=1}^{r} V_{\left(j_{\ell}, j_{\ell}^{*}\right)}^{*}\right) \otimes\left(\bigotimes_{\ell=1}^{s} V_{\left(k_{\ell}, k_{\ell}^{*}\right)}\right)
$$

Here the identification $\operatorname{Hom}_{S O(4)}(U, W) \cong \operatorname{Hom}_{S O(4)}\left(\mathbb{C}, U^{*} \otimes W\right)$ was used as well as the fact that $\overline{H_{p q}^{(j)}}=H_{p q}^{\left(j^{*}\right)}$ and $V_{\left(j, j^{*}\right)}^{*} \cong V_{j}^{*} \otimes V_{j}^{* *}$. The index pairs $\left(p_{\ell} q_{\ell}\right)$ therefore correspond to the tensor factors $V_{\left(j_{\ell}, j_{\ell}^{*}\right)}$ of the domain of $I$ while the $\left(m_{\ell} n_{\ell}\right)$ belong to the image $V_{\left(k_{\ell}, k_{\ell}^{*}\right)}$.

Lemma 3.7. The map $I$ defined by $(\underline{3.6})$ is a morphism of representations of $S O(4)$.

Proof. The action of $S O(4)$ is given by the action of $S U(2) \times S U(2)$ in (2.28). If we write $S U(2) \cong S^{3}$ for the range of the integral,

$$
I_{\left(m_{1} n_{1}\right) \ldots ;\left(p_{1} q_{1}\right) \ldots}:=\int_{S U(2)} t_{p_{1} q_{1}}^{\left(j_{1}^{*}\right)}(g) \cdots t_{p_{r} q_{r}}^{\left(j_{r}^{*}\right)}(g) t_{m_{1} n_{1}}^{\left(k_{1}\right)}(g) \cdots t_{m_{s} n_{s}}^{\left(k_{s}\right)}(g) d g
$$

this action corresponds to $S U(2)$ left-right translation of all function arguments which is an invariance of the Haar measure of $S U(2)$.

\subsection{Definition of the model}

The Barrett-Crane model can now be defined by an expression similar to the dual of lattice gauge theory (Theorem 3.4) in which the Haar intertwiner is replaced by a pair of BarrettCrane intertwiners, one associated to each vertex of the given edge. These two Barrett-Crane intertwiners are 'independent' which corresponds to the first version of the model proposed in 倠.

Definition 3.8. Let $(V, E, F)$ denote a finite 2-complex with cyclic structure, let $\widehat{w}_{j} \in \mathbb{C}$, $j \in \frac{1}{2} \mathrm{~N}_{0}$, be a face amplitude and

$$
\mathcal{A}^{(e)}(\underbrace{j_{f}, \ldots}_{f \in \delta e}) \in \mathbb{C}
$$




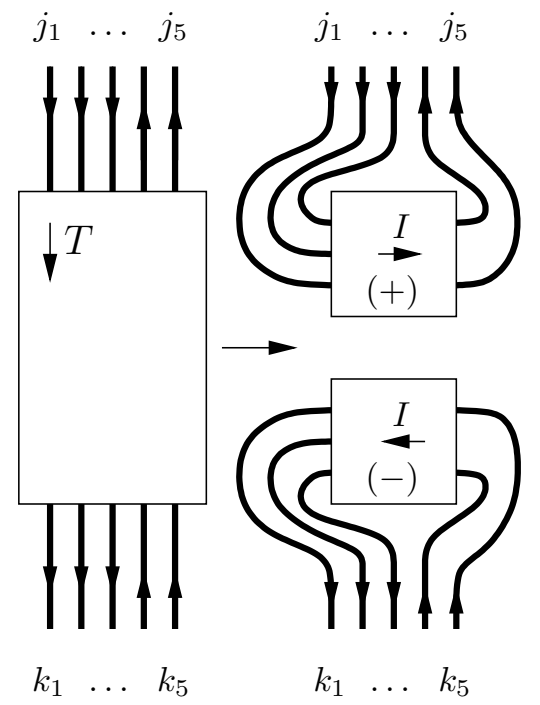

(a)

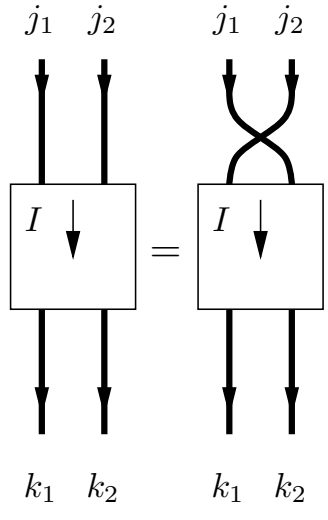

(b)

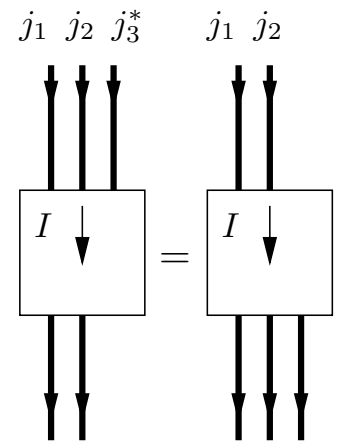

$k_{1} k_{2}$

Figure 4: (a) Replacing the $S O(4)$ Haar intertwiner by a pair of Barrett-Crane intertwiners (3.6). The lines labelled $j$ represent balanced irreducible representations $V_{\left(j, j^{*}\right)}$ of $S O(4)$. (b) Commutativity of the algebra of spherical functions. (c) Relation of the conjugate spherical function with the dual representation.

$j_{f} \in \frac{1}{2} N_{0}$, an edge amplitude. The Barrett-Crane model is defined by the partition function,

$$
\begin{aligned}
Z= & \left(\prod_{f \in F} \sum_{j_{f} \in \frac{1}{2} \mathbb{N}_{0}}\right)\left(\prod_{f \in F} \widehat{w}_{j_{f}}\right)\left(\prod_{e \in E} \mathcal{A}^{(e)}(\underbrace{j_{f}, \ldots}_{f \in \delta e})\right)\left(\prod_{f \in F} \prod_{v \in f_{0}} \sum_{n(f, v)=1}^{2 j_{f}+1} \sum_{m(f, v)=1}^{2 j_{f}+1}\right) \\
& \times \prod_{e \in E} \underbrace{(+, e)}_{f \in e_{+}} \underbrace{\left(n\left(f, \partial_{+} e\right) m\left(f, \partial_{+} e\right)\right) \ldots ;}_{f \in e_{-}} \underbrace{\left(n\left(f, \partial_{+} e\right) m\left(f, \partial_{+} e\right)\right) \ldots}_{f \in e_{-}} \cdot \underbrace{\left.I_{(n, e)}^{(-, e)}\left(f, \partial_{-} e\right) m\left(f, \partial_{-} e\right)\right) \ldots ;\left(n\left(f, \partial_{-} e\right) m\left(f, \partial_{-} e\right)\right) \ldots}_{f \in e_{+}} .
\end{aligned}
$$

Here $I_{\ldots}^{(+, e)}$ and $I_{\ldots}^{(-, e)}, e \in E$, denote the coefficients of the Barrett-Crane intertwiners (3.6),

$$
\begin{aligned}
I^{(+, e)}: & \bigotimes_{f \in e_{-}} V_{\left(j_{f}, j_{f}^{*}\right)} \rightarrow \bigotimes_{f \in e_{+}} V_{\left(j_{f}, j_{f}^{*}\right)}, \\
I^{(-, e)}: & \bigotimes_{f \in e_{+}} V_{\left(j_{f}, j_{f}^{*}\right)} \rightarrow \bigotimes_{f \in e_{-}} V_{\left(j_{f}, j_{f}^{*}\right)},
\end{aligned}
$$

where $V_{\left(j, j^{*}\right)}=V_{j} \otimes V_{j}^{*}$ are balanced irreducible representations of $S O(4)$ whose right factors have been dualized.

Remark 3.9. $\quad$ 1. The partition function $(3.10)$ can be described in words as follows. The faces $f \in F$ are coloured in all possible ways with representations $V_{\left(j, j^{*}\right)}=V_{j} \otimes V_{j}^{*}, j \in$ $\frac{1}{2} \mathrm{~N}_{0}$, of $S O(4)$. There is an amplitude $\widehat{w}_{j_{f}}$ for each face and an amplitude $\mathcal{A}^{(e)}\left(j_{f}, \ldots\right)$ 


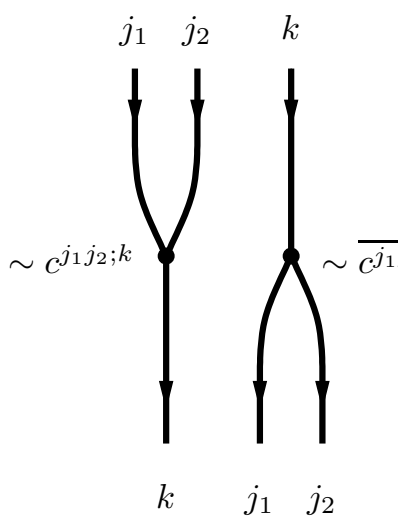

(a)

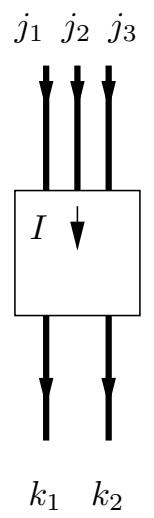

(c)

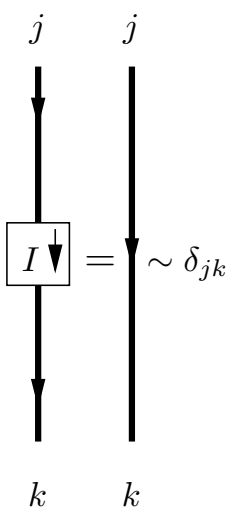

(d)

Figure 5: (a) Trivalent vertices represent the recoupling coefficients of the spherical functions. (b) Commutativity of the algebra. (c) Linearity of the integral. (d) The orthogonality relation for spherical functions.

for each edge which can depend on the representations at all faces $f \in \delta e$. Each edge $e \in E$ is labelled with a pair of Barrett-Crane intertwiners $I^{(+, e)}$ and $I^{(-, e)}$, each one associated to one vertex, and the vector indices (here pairs $(m, n)$ for $\left.V_{j} \otimes V_{j}^{*}\right)$ are contracted precisely as in the spin foam model dual to lattice gauge theory (Theorem 3.4 and Figure 3(a)). Figure $1(\mathrm{a})$ visualizes the replacement of the $S O(4)$ Haar intertwiner $T$ by the pair of Barrett-Crane intertwiners $I$. The contraction of the indices in the Barrett-Crane model is illustrated in Figure 3(b). In this figure, we denote the BarrettCrane intertwiners by the full dots, a simplification which we justify below.

2. The face amplitude is usually chosen to be $\widehat{w}_{j}=\operatorname{dim} V_{\left(j, j^{*}\right)}=(2 j+1)^{2}$ while there are various edge amplitudes proposed in the literature [5, 15 [17]. We leave the amplitudes unspecified here and discuss the question of the convergence of the partition function later in Section 4.4 .

3. For all balanced irreducible representations $V_{j} \otimes V_{j}$ of $S U(2) \times S U(2)$, we have identified the tensor factor corresponding to the right $S U(2)$ by its dual. Our model still agrees with the original proposal [4] since the corresponding isomorphism appears in each representation of $S O(4)$, i.e. in each line in Figure 3(b), together with its inverse in such a way that they cancel. We have, however, obtained a considerable technical advantage with this construction as we can now use the spherical functions of Section 2.5 which agree with the representation functions of $S U(2)$.

\subsection{Properties of the Barrett-Crane intertwiner}

In this section, we comment on a number of properties of the Barrett-Crane intertwiner (3.6) and translate some results of 18 into our language. This intertwiner is visualized by the box diagrams labelled $I$ in Figure 4 (a). Each line carries a label $j \in \frac{1}{2} \mathbb{N}_{0}$ and symbolizes the representation $V_{j} \otimes V_{j}^{*}$ of $S U(2) \times S U(2)$ if the arrow points down and the dual representation 


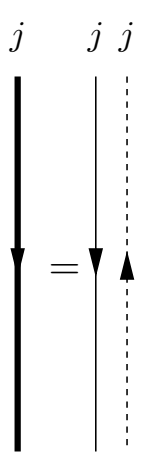

(a)

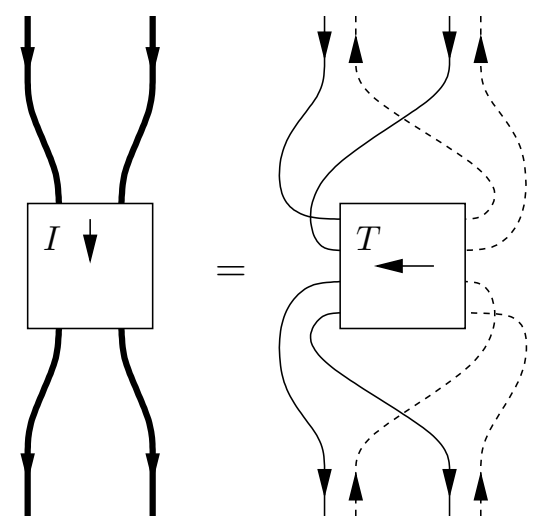

(b)

Figure 6: (a) Expressing representations $V_{\left(j, j^{*}\right)}=V_{j} \otimes V_{j}^{*}$ of $S O(4)$ as a tensor product of representations of $S U(2)$. The tensor factor corresponding to the right $S U(2)$ is drawn as a dotted line. (b) The Barrett-Crane intertwiner as an $S U(2)$ integral (3.13a).

$\left(V_{j} \otimes V_{j}^{*}\right)^{*}$ if the arrow points up.

Commutativity of the algebra $C_{\text {alg }}\left(S^{3}\right)$ gives rise to rules such as the one depicted in Figure 4(b). If we use $\overline{H_{m n}^{(j)}}=H_{m n}^{\left(j^{*}\right)}$ in the integrand, we obtain rules as in Figure $4(\mathrm{c})$. The lines can therefore be freely moved around the diagram, and what matters is only whether the arrows point towards or away from the box. Therefore, in Figure 3(b), it is sufficient to draw full dots with incoming or outgoing lines for the Barrett-Crane intertwiners. Note that each arrow can be reversed if at the same time the representation label $j$ is replaced by its dual $j^{*}$.

We can make use of the fact that $C_{\text {alg }}\left(S^{3}\right)$ forms an algebra for which the spherical functions provide a basis (Corollary 2.8), therefore,

$$
\begin{aligned}
& H_{m_{1} n_{1}}^{\left(j_{1}\right)} \cdot H_{m_{2} n_{2}}^{\left(j_{2}\right)}=\sum_{k \in \frac{1}{2} \mathbb{N}_{0}} \sum_{p, q=1}^{2 k+1} \sqrt{\frac{2 k+1}{\left(2 j_{1}+1\right)\left(2 j_{2}+1\right)}} \cdot c_{m_{1} n_{1}, m_{2} n_{2} ; p q}^{\left(j_{1} j_{2} ; k\right)} \cdot H_{p q}^{(k)}, \\
& \overline{H_{m_{1} n_{1}}^{\left(j_{1}\right)}} \cdot \overline{H_{m_{2} n_{2}}^{\left(j_{2}\right)}}=\sum_{k \in \frac{1}{2} \mathbb{N}_{0}} \sum_{p, q=1}^{2 k+1} \sqrt{\frac{2 k+1}{\left(2 j_{1}+1\right)\left(2 j_{2}+1\right)}} \cdot \overline{c_{m_{1} n_{1}, m_{2} n_{2} ; p q}^{\left(j_{1} j_{2} ; k\right)} \cdot \overline{H_{p q}^{(k)}}},
\end{aligned}
$$

for $j_{1}, j_{2} \in \frac{1}{2} \mathbb{N}_{0}$ and $1 \leq m_{\ell}, n_{\ell} \leq 2 j_{\ell}+1$, with uniquely determined coefficients $c_{m_{1} n_{1}, m_{2} n_{2} ; p q}^{\left(j_{1} j_{2} ; k\right)}$ $\mathbb{C}$. This is illustrated in Figure $5(a)$. Rules such as the one depicted in diagram (b) follow from the commutativity of the algebra. Linearity of the integral implies rules as in diagram (c), and the orthogonality of spherical functions (2.33) finally leads to diagram (d).

These rules can be used in order to decompose the Barrett-Crane intertwiner into a tree diagram involving only three-valent vertices [18]. For each line connecting two vertices, the corresponding indices are contracted, and we sum over all representations of the form $V_{j} \otimes V_{j}^{*}$ for each internal edge. Obviously, all possible decompositions into trees evaluate to 


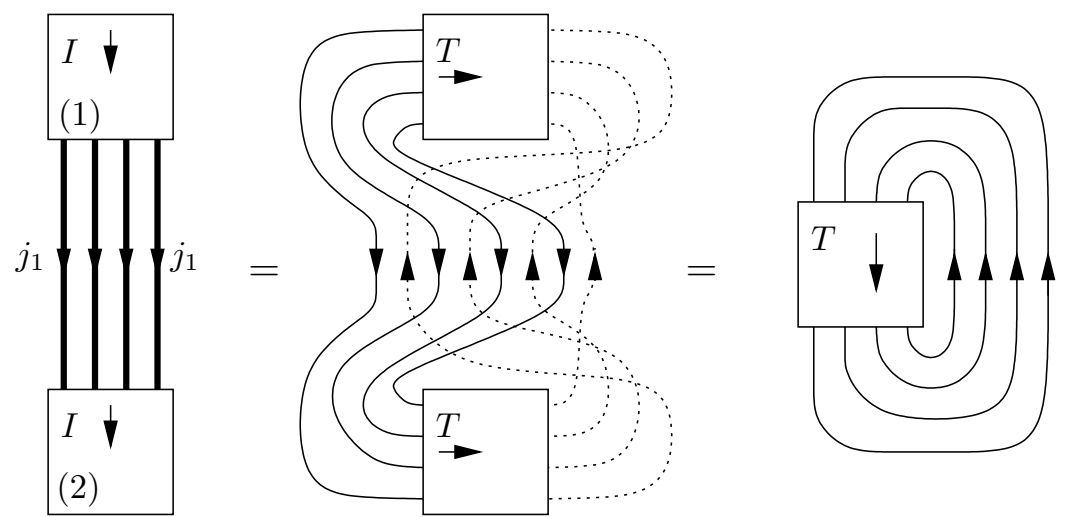

Figure 7: Calculation in order to determine the normalization of the BarrettCrane intertwiner (3.6). The Barrett-Crane intertwiner is denoted by the boxes labelled $I$ while the boxes $T$ represent the $S U(2)$ Haar intertwiner. In the first step, we have used (3.13a) (Figure $6(\mathrm{~b})$ ), and in the second step the projector property (2.21) of the Haar intertwiner.

the same intertwiner (3.6) [18]. This is the property that was originally required in [4, 27]. We emphasize that we here use the intertwiner in the form involving the spherical functions $H_{m n}^{(j)}$ whose orthogonality relation (2.33) involves a denominator of $2 j+1$. The normalized basis vectors of $C_{\mathrm{alg}}\left(S^{3}\right)$ are therefore $\sqrt{2 j+1} \cdot H_{m n}^{(j)}$.

Furthermore, we can employ the identification of the $S^{3}$-integral of spherical functions with the $S U(2)$-integral of representation functions (Proposition 2.9) and express the BarrettCrane intertwiner as an $S U(2)$ integral,

$$
\begin{aligned}
& \int_{S^{3}} \overline{H_{p_{1} q_{1}}^{\left(j_{1}\right)}(x) \cdots H_{p_{r} q_{r}}^{\left(j_{r}\right)}(x)} \cdot H_{m_{1} n_{1}}^{\left(k_{1}\right)}(x) \cdots H_{m_{s} n_{s}}^{\left(k_{s}\right)}(x) d x \\
= & \int_{S U(2)} t_{p_{1} q_{1}}^{\left(j_{1}^{*}\right)}(g) \cdots t_{p_{r} q_{r}}^{\left(j_{r}^{*}\right)}(g) \cdot t_{m_{1} n_{1}}^{\left(k_{1}\right)}(g) \cdots t_{m_{s} n_{s}}^{\left(k_{s}\right)}(g) d g,
\end{aligned}
$$

so that we find

$$
I_{\left(m_{1} n_{1}\right) \ldots ;\left(p_{1} q_{1}\right) \ldots}=T_{p_{1} \ldots m_{1} \ldots ; q_{1} \ldots n_{1} \ldots}
$$

where $T$ is the $S U(2)$ Haar intertwiner,

$$
T:\left(\bigotimes_{\ell=1}^{s} V_{k_{\ell}}\right) \otimes\left(\bigotimes_{\ell=1}^{r} V_{j_{\ell}}^{*}\right) \rightarrow\left(\bigotimes_{\ell=1}^{s} V_{k_{\ell}}\right) \otimes\left(\bigotimes_{\ell=1}^{r} V_{j_{\ell}}^{*}\right) .
$$

This relation is shown in Figure 6. In diagram (a), the representations $V_{\left(j, j^{*}\right)} \cong V_{j} \otimes V_{j}^{*}$ of $S O(4)$ are split into their tensor factors as representations of $S U(2) \times S U(2)$ where we have dualized all right factors. Diagram (b) visualizes (3.13). 
Finally, we show that the Barrett-Crane intertwiner of Definition 3.6 agrees with the unnormalized intertwiner in the terminology of 15]. Therefore we consider the intertwiners $I^{(1)}: \mathbb{C} \rightarrow V_{\left(j_{1}, j_{1}^{*}\right)} \otimes \cdots \otimes V_{\left(j_{4}, j_{4}^{*}\right)}$ and $I^{(2)}: V_{\left(j_{1}, j_{1}^{*}\right)} \otimes \cdots \otimes V_{\left(j_{4}, j_{4}^{*}\right)} \rightarrow \mathbb{C}$ for a four-fold tensor product and compute

$$
\begin{aligned}
& \sum_{m_{1}, n_{1}=1}^{2 j_{1}+1} \cdots \sum_{m_{4}, n_{4}=1}^{2 j_{4}+1} I_{\left(m_{1} n_{1}\right) \cdots\left(m_{4} n_{4}\right)}^{(1)} \cdot I_{\left(m_{1} n_{1}\right) \ldots\left(m_{4} n_{4}\right)}^{(2)} \\
= & \int_{S U(2)} \chi^{\left(j_{1}\right)}(g) \chi^{\left(j_{2}\right)}(g) \chi^{\left(j_{3}\right)}(g) \chi^{\left(j_{4}\right)}(g) d g \\
= & \operatorname{dim} \operatorname{Hom}_{S U(2)}\left(V_{j_{1}} \otimes V_{j_{2}}, V_{j_{3}} \otimes V_{j_{4}}\right) .
\end{aligned}
$$

This calculation is illustrated in Figure 7. A comparison shows that our Barrett-Crane intertwiner agrees with the unnormalized one of [15]. If we wish to use a different normalization and multiply our intertwiner by a factor, we can always absorb this factor into the edge amplitude $\mathcal{A}^{(e)}$. We emphasize that the Barrett-Crane intertwiner for given representations is unique only up to normalization [27], but that the relative weight of different representations is not determined. We demonstrate in Section 4.4 that the physical properties of the model are affected by the choice of normalization.

\section{Dual variables for the Barrett-Crane model}

\subsection{The duality transformation}

We can employ the integral presentation of the Barrett-Crane intertwiner (3.6) in order to introduce $S^{3}$-variables into the Barrett-Crane model. It is possible to derive a transformation similar to the reversed direction of the duality transformation for lattice gauge theory in order to promote the new $S^{3}$-values to the configuration variables of the partition function.

First we make use of the integral presentation of the Barrett-Crane intertwiner,

$$
\begin{aligned}
& \underbrace{(+, e)}_{f \in e_{+}} \underbrace{n\left(f, \partial_{+} e\right) m\left(f, \partial_{+} e\right) \ldots ;}_{f \in e_{-}} \underbrace{n\left(f, \partial_{+} e\right) m\left(f, \partial_{+} e\right) \ldots}_{f \in e_{-}} \cdot \underbrace{I_{n\left(f, \partial_{-} e\right) m\left(f, \partial_{-} e\right) \ldots}^{(-, e)}}_{f \in e_{+}} \underbrace{n\left(f, e^{\prime}\right.}_{\underbrace{n\left(f, \partial_{-} e\right) m\left(f, \partial_{-} e\right) \ldots}} \\
& =\int_{S U(2)} \underbrace{t_{n\left(f, \partial_{+} e\right) m\left(f, \partial_{+} e\right)}^{\left(j_{f}\right)}(g) \cdots}_{f \in e_{+}} \underbrace{t_{n\left(f, \partial_{+} e\right) m\left(f, \partial_{+} e\right)}^{\left(j_{f}^{*}\right)}(g) \cdots}_{f \in e_{-}} d g \\
& \times \int_{S U(2)} \underbrace{t_{n\left(f, \partial_{-} e\right) m\left(f, \partial_{-} e\right)}^{\left(j_{f}\right)}(g) \cdots}_{f \in e_{-}} \underbrace{t_{n\left(f, \partial_{-} e\right) m\left(f, \partial_{-} e\right)}^{\left(j_{f}^{*}\right)}(g) \cdots}_{f \in e_{+}} d g .
\end{aligned}
$$


We insert this expression into the partition function (3.10) and move all integrals to the left,

$$
\begin{aligned}
& Z=\left(\prod_{e \in E_{S U}(2)} \int_{S g_{e}^{(+)}} \int_{S U(2)} d g_{e}^{(-)}\right)\left(\prod_{f \in F} \sum_{j_{f} \in \frac{1}{2} \mathbb{N}_{0}}\right)\left(\prod_{f \in F} \widehat{w}_{j_{f}}\right)\left(\prod_{e \in E} \mathcal{A}^{(e)}(\underbrace{j_{f}, \ldots}_{f \in \delta e})\right) \\
& \times\left(\prod_{f \in F} \prod_{v \in f_{0}} \sum_{n(f, v)=1}^{2 j_{f}+1} \sum_{m(f, v)=1}^{2 j_{f}+1}\right) \\
& \times \prod_{e \in E}\left[\left(\prod_{f \in e_{-}} t_{m\left(f, \partial_{+} e\right) n\left(f, \partial_{+} e\right)}^{\left(j_{f}\right)}\left(g_{e}^{(+)^{-1}}\right) \cdot t_{n\left(f, \partial_{-} e\right) m\left(f, \partial_{-} e\right)}^{\left(j_{f}\right)}\left(g_{e}^{(-)}\right)\right)\right. \\
& \left.\left(\prod_{f \in e_{+}} t_{n\left(f, \partial_{+} e\right) m\left(f, \partial_{+} e\right)}^{\left(j_{f}\right)}\left(g_{e}^{(+)}\right) \cdot t_{m\left(f, \partial_{-} e\right) n\left(f, \partial_{-} e\right)}^{\left(j_{f}\right)}\left(g_{e}^{(-)^{-1}}\right)\right)\right] .
\end{aligned}
$$

Here we have also employed the antipode (2.8) in order to remove dual representations in favour of inverse group elements.

Keeping the similarity with lattice gauge theory in mind, we try to factor the integrand into one contribution for each face. Therefore, we sort the products in the above expression by face rather than by edge. This step is almost entirely hidden in our notation,

$$
\begin{aligned}
Z= & \left(\prod_{e \in E_{S U}(2)} \int_{S g_{e}^{(+)}} \int_{S U(2)} d g_{e}^{(-)}\right)\left(\prod_{f \in F} \sum_{j_{f} \in \frac{1}{2} \mathbb{N}_{0}}\right)\left(\prod_{f \in F} \widehat{w}_{j_{f}}\right)\left(\prod_{e \in E} \mathcal{A}^{(e)}(\underbrace{j_{f}, \ldots}_{f \in \delta e})\right) \\
\times & \left(\prod_{f \in F} \prod_{v \in f_{0}} \sum_{n(f, v)=1}^{2 j_{f}+1} \sum_{m(f, v)=1}^{2 j_{f}+1}\right) \\
\times & \prod_{f \in F}\left[\left(\prod_{e \in f_{-}} t_{m\left(f, \partial_{+} e\right) n\left(f, \partial_{+} e\right)}^{\left(j_{f}\right)}\left(g_{e}^{(+)^{-1}}\right) \cdot t_{n\left(f, \partial_{-} e\right) m\left(f, \partial_{-} e\right)}^{\left(j_{f}\right)}\left(g_{e}^{(-)}\right)\right)\right. \\
& \left.\left.\left(\prod_{e \in f_{+}} t_{n\left(f, \partial_{+} e\right) m\left(f, \partial_{+} e\right)}^{\left(j_{f}\right)}\left(g_{e}^{(+)}\right) \cdot t_{m\left(f, \partial_{-} e\right) n\left(f, \partial_{-} e\right)}^{\left(j_{f}\right)}\left(g_{e}^{(-)}\right)^{-1}\right)\right)\right] .
\end{aligned}
$$

The structure of the integrand becomes clearer if we re-introduce the cyclic ordering of edges in the boundary of each face,

$$
\begin{aligned}
Z= & \left(\prod_{e \in E} \int_{S U(2)} d g_{e}^{(+)} \int_{S U(2)} d g_{e}^{(-)}\right)\left(\prod_{f \in F} \sum_{j_{f} \in \frac{1}{2} \mathbb{N}_{0}}\right)\left(\prod_{e \in E} \mathcal{A}^{(e)}(\underbrace{j_{f}, \ldots}_{f \in \delta e})\right) \\
\times & \prod_{f \in F}\left[\widehat{w}_{j_{f}} \prod_{v \in f_{0}} \sum_{n(v)=1}^{2 j_{f}+1} \sum_{m(v)=1}^{2 j_{f}+1}\right. \\
& \left.\prod_{k=1}^{N(f)} t_{m\left(\partial_{-\varepsilon_{k}} \partial_{k} f\right) n\left(\partial_{-\varepsilon_{k}} \partial_{k} f\right)}\left(g_{\partial_{k} f}^{\left(\varepsilon_{k} f\right)^{-1}}\right) \cdot t_{n\left(\partial_{\varepsilon_{k}} \partial_{k} f\right) m\left(\partial_{\varepsilon_{k}} \partial_{k} f\right)}\left(g_{\partial_{k} f}^{\left(\varepsilon_{k} f\right)}\right)\right] .
\end{aligned}
$$

Recall that the cyclic structure of the edges around a face is described by the conditions $(2.36)$. We notice that in the last product in (4.4), both indices of the second $t_{n m}^{(j)}$ of each factor are contracted with the indices of the first $t_{n m}^{(j)}$ of the following factor and so on. 
We therefore shift the factors of the product by a single $t_{n m}^{(j)}$. Now the indices $n(v)$ and $m(v)$ occur only in a single factor,

$$
\begin{aligned}
Z= & \left(\prod_{e \in E_{S U}(2)} \int_{f} d g_{e}^{(+)} \int_{S U(2)} d g_{e}^{(-)}\right)\left(\prod_{f \in F} \sum_{j_{f} \in \frac{1}{2} \mathbb{N}_{0}}\right)\left(\prod_{e \in E} \mathcal{A}^{(e)}(\underbrace{j_{f}, \ldots}_{f \in \delta e})\right) \\
& \times \prod_{f \in F}\left[\widehat{w}_{j_{f}} \prod_{v \in f_{0}} \sum_{n=1}^{2 j_{f}+1} \sum_{m=1}^{2 j_{f}+1} t_{n m}^{\left(j_{f}\right)}\left(g_{v_{+}(f)}^{(+)}\right) t_{m n}^{\left(j_{f}\right)}\left(g_{v_{-}(f)}^{(-)}{ }^{-1}\right)\right],
\end{aligned}
$$

where $v_{+}(f)$ denotes the edge in the boundary of the face $f \in F$ for which $v=\partial_{+} e$, etc.. The last expression involves just a group character. We arrive at the following result.

Theorem 4.1 (Dual variables). Let $(V, E, F)$ be a finite 2-complex with cyclic structure, let $\widehat{w}_{j} \in \mathbb{C}, j \in \frac{1}{2} \mathbb{N}_{0}$, be a face amplitude and $\mathcal{A}^{(e)}\left(j_{f}, \ldots\right)$ denote an edge amplitude, $j_{f} \in \frac{1}{2} \mathbb{N}_{0}$. The partition function of the Barrett-Crane model (3.10) is equal to the following expression,

$$
\begin{aligned}
Z= & \left(\prod_{e \in E_{S U}(2)} \int_{S g_{e}} d+\int_{S U(2)} d g_{e}^{(-)}\right)\left(\prod_{f \in F} \sum_{j_{f} \in \frac{1}{2} \mathbb{N}_{0}}\right)\left(\prod_{e \in E} \mathcal{A}^{(e)}(\underbrace{j_{f}, \ldots}_{f \in \delta e})\right) \\
& \times \prod_{f \in F}\left[\widehat{w}_{j_{f}} \prod_{v \in f_{0}} \chi^{\left(j_{f}\right)}\left(g_{v_{+}(f)}^{(+)} \cdot g_{v_{-}(f)}^{(-)}\right)\right] .
\end{aligned}
$$

If the edge amplitude factors into one contribution for each face attached to the edge,

$$
\mathcal{A}^{(e)}(\underbrace{j_{f}, \ldots}_{f \in \delta e})=\prod_{f \in \delta e} \widehat{v}_{j_{f}},
$$

$\widehat{v}_{j_{f}} \in \mathbb{C}$, this can be written as

$$
Z=\left(\prod_{e \in E_{S U(2)}} \int_{S U(2)} d g_{e}^{(+)} \int_{S g_{e}} d g_{e}^{(-)}\right) \prod_{f \in F} f\left(g_{\partial_{1} f}^{(+)}, g_{\partial_{1} f}^{(-)}, g_{\partial_{2} f}^{(+)}, g_{\partial_{2} f}^{(-)}, \ldots, g_{\partial_{N(f)} f}^{(+)}, g_{\partial_{N(f)} f}^{(-)}\right),
$$

where

$$
f\left(g_{1}^{(+)}, g_{1}^{(-)}, \ldots\right)=\sum_{j \in \frac{1}{2} \mathbb{N}_{0}} \widehat{w}_{j} \widehat{v}_{j}^{|\partial f|} \chi^{(j)}\left(g_{2}^{(+)} g_{1}^{(-)^{-1}}\right) \chi^{(j)}\left(g_{3}^{(+)} g_{2}^{(-)^{-1}}\right) \cdots \chi^{(j)}\left(g_{1}^{(+)} g_{k}^{(-)^{-1}}\right) .
$$

Remark 4.2. 1. The partition function (4.6) describes a system in Statistical Mechanics whose variables are pairs of $S U(2)$ values $\left(g_{e}^{(+)}, g_{e}^{(-)}\right)$attached to each edge $e \in E$. For a generic edge amplitude $\mathcal{A}^{(e)}$, the Boltzmann weight under the partition sum forms a non-local expression.

2. If the edge amplitude factorizes into one contribution per face, the Boltzmann weight factorizes into a product over all faces (4.8a). The factor $f\left(g_{1}^{(+)}, g_{1}^{(-)}, \ldots, g_{k}^{(+)}, g_{k}^{(-)}\right)$ for each face $f \in F$ depends on the group elements at all edges in the boundary of 


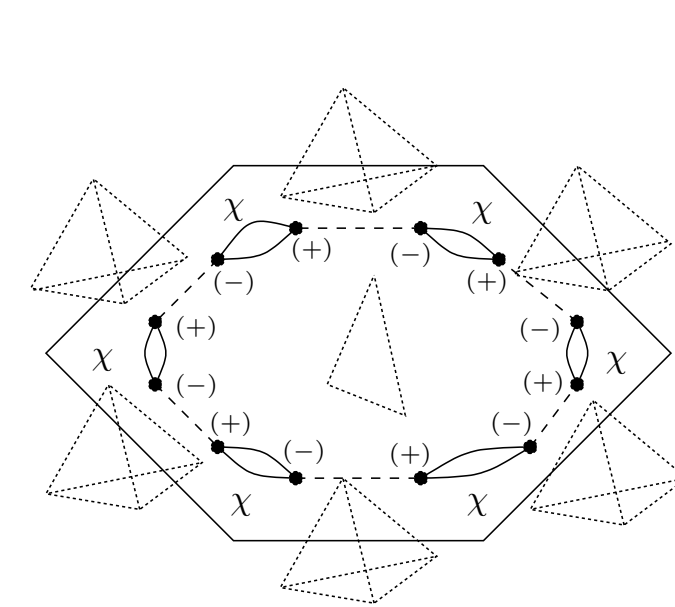

(a)

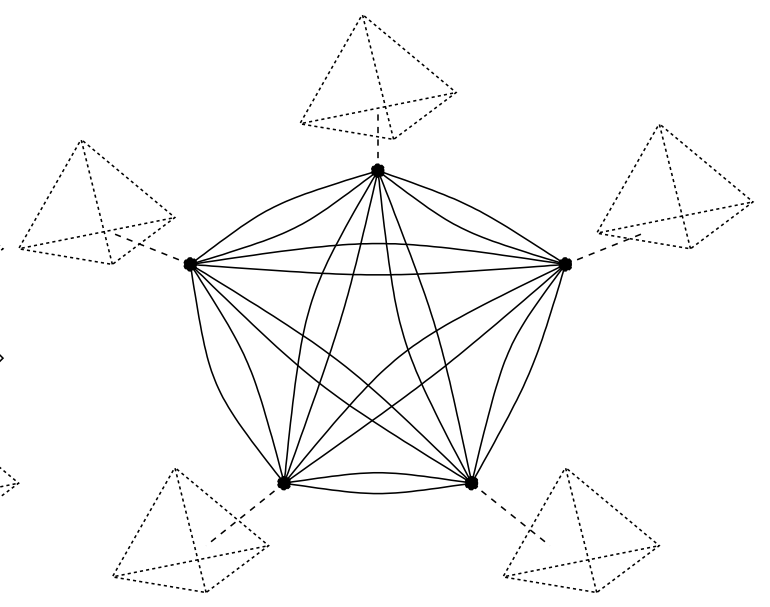

(b)

Figure 8: (a) The tetrahedra sharing a common triangle ('exploded' drawing). The solid polygon denotes the corresponding dual face. A pair of $S^{3}$-variables (dots) is associated to each tetrahedron. The interaction term for this triangle depends on some characters $(\chi)$ calculated for each pair of neighbouring tetrahedra (pairs of solid lines). However, each tetrahedron contributes with different $S^{3}$ values $(+)$ or $(-)$ depending on the neighbour. There is therefore some parallel transport associated to the tetrahedra (dashed lines). (b) The five tetrahedra in the boundary of a given four-simplex. This diagram visualizes all characters that are calculated for the weights associated to the various triangles. Each pair of lines belongs to one of the 10 triangles involved in the four-simplex and coincides with a pair of lines in diagram (a) when (a) is drawn for that triangle.

$f$, but only through the characters $\chi^{(j)}\left(g_{\ell+1}^{(+)} \cdot g_{\ell}^{(-)^{-1}}\right)$ for each pair of group elements $\left(g_{\ell+1}^{(+)}, g_{\ell}^{(-)}\right)$that are associated to neighbouring edges. Although this interaction is considerably more complicated than just a nearest neighbour interaction, it is still local in the sense that there is one contribution for each face, and the interaction term for a given face relies only on data associated to the nearby edges.

3. We discuss the properties of the Boltzmann weight $f\left(g_{1}^{(+)}, g_{1}^{(-)}, \ldots\right)$ and the possible choices for the amplitudes $w_{j}$ and $\mathcal{A}^{(e)}$ in Section 4.4.

4. Equation (4.6) is an intermediate step in the transformation in which both types of variables are present, the old representation numbers and the new group variables.

\subsection{Geometric interpretation}

In this section, we restrict ourselves to the case in which the interactions are local, i.e. to the situation in which the edge amplitude factorizes according to (4.7). We consider a 2complex $(V, E, F)$ that is dual to a given triangulation of a four-manifold. For more general 2-complexes dual to a generic cellular decomposition, the interpretation has to be generalized 
accordingly.

Using the identification $S U(2) \cong S^{3}$, the partition function 4.8a suggests the following geometric interpretation. The variables of the model are the pairs of $S^{3}$-values associated to the tetrahedra of the triangulation. An oriented tetrahedron, embedded in $\mathbb{R}^{4}$, spans an oriented hyperplane which can be parameterized by its (normalized) normal vector, i.e. by a point in $S^{3} \subset \mathbb{R}^{4}$.

The partition function contains an interaction term $f\left(g_{1}^{(+)}, g_{1}^{(-)}, \ldots\right)$ for each triangle. The interaction depends on the $S^{3}$-values for all tetrahedra that contain the triangle in their boundaries. Recall that the characters of $S U(2)$ are given by

$$
\chi^{(j)}(g)=\frac{\sin ((2 j+1) \varphi)}{\sin (\varphi)}
$$

where $\varphi$ denotes the polar angle on $S^{3} \cong S U(2)$. The interaction therefore depends only on the relative angles between the normal vectors of each pair of neighbouring tetrahedra, the so-called dihedral angles. Two tetrahedra are neighbours with respect to a given triangle if they are contained in the boundary of the same 4-simplex (this is the same as saying that on the dual 2-complex, two neighbouring edges contain a common vertex, but expressed in the language of the original triangulation).

However, there is the complication that each tetrahedron carries two $S^{3}$-variables, $g_{e}^{(+)}$ and $g_{e}^{(-)}$. For a given triangle, each of these values is involved in the interaction term: the first variable, $g_{e}^{(+)}$, in association with one neighbour and the second variable, $g_{e}^{(-)}$, with the other one, see Figure 8(a). The tetrahedron dual to $e$ therefore contributes with different $S^{3}$-values $g_{e}^{(+)}$and $g_{e}^{(-)}$depending on the four-simplex in which the interaction takes place. It is thus plausible to conjecture that there is some parallel transport associated to the tetrahedra which contains the information of how $g_{e}^{(-)}$is rotated to $g_{e}^{(+)}$when we transit from one four-simplex along a tetrahedron to a neighbouring four-simplex. The obvious candidate for the parallel transport is the transitive $S O(4)$-action on $S^{3}$ for which the $S O(4)$ element is, however, only defined up to an element in the stabilizer of a given point on $S^{3}$. In Section 4.3, we reconstruct the $S O(4)$ parallel transport, and our intuition about the ambiguity due to the stabilizer is shown to be correct.

The weight (4.8b) resembles the character decomposition of a function on $S U(2) \times S U(2)$ where the character decomposition can be understood as a generalization of Fourier expansion. Because of the geometric picture, the $S^{3}$-variables have a natural interpretation円 as variables conjugate to the representations labelling the faces - conjugate in the sense of the quantum mechanical uncertainty relation.

If we suppose that the partition function can be interpreted as the trace over a suitable Hilbert space, there are two pictures for the Barrett-Crane model in analogy to position and momentum basis in Quantum Mechanics: In the original formulation, the partition function is a sum over all possible quantized areas associated to the triangles where the areas are determined by the balanced irreducible representation of $S O(4)[5,14]$. If we are in a quantum state with definite areas of the triangles, the directions of the tetrahedra in $\mathbb{R}^{4}$ which are represented by the $S^{3}$-variables and the corresponding dihedral angles, would be maximally uncertain.

\footnotetext{
${ }^{1}$ This interpretation was suggested by D. Oriti. Further work on the geometric interpretation at the classical and at the quantum level is in progress 28 .
} 
In addition to this 'area basis' we also have a 'direction basis' which is given by the dual partition sum. The dual partition sum is the integral over all possible $S^{3}$-directions of the tetrahedra. In a quantum state with definite directions, however, we would expect a maximal uncertainty of the quantized areas assigned to the triangles.

\subsection{The gauge theory picture}

We have conjectured above that it is possible to find the parallel transports of an $S O(4)$ connection which, for each edge $e \in E$, map the first $S^{3}$-variable $g_{e}^{(-)}$to the second one $g_{e}^{(+)}$. This would mean that the Barrett-Crane model has some properties of an $S O(4)$ gauge theory and therefore enjoys the corresponding local symmetry. A suitable notion of local $S O(4)$ symmetry is the invariance of the Boltzmann weight under a transformation which takes an arbitrary $S O(4)$ element for each vertex $v \in V$ and transforms each edge variable $\left(g_{e}^{(+)}, g_{e}^{(-)}\right)$depending on the group elements that have been chosen for the two vertices.

Proposition 4.3. Let $h: V \rightarrow S O(4), v \mapsto\left(h_{v}, \widetilde{h}_{v}\right)$ define a generating function. Here $\left(h_{v}, \widetilde{h}_{v}\right) \in S U(2) \times S U(2)$ represents a class of $S O(4) \cong(S U(2) \times S U(2)) / \mathbb{Z}_{2}$. The BarrettCrane model (4.8a) is invariant under the local transformations,

$$
\begin{gathered}
g_{e}^{(+)} \mapsto h_{\partial_{+} e} \cdot g_{e}^{(+)} \cdot \widetilde{h}_{\partial_{+} e}^{-1}, \\
g_{e}^{(-)} \mapsto h_{\partial_{-} e} \cdot g_{e}^{(-)} \cdot \widetilde{h}_{\partial_{-} e}^{-1} .
\end{gathered}
$$

By Proposition 2.7, this transformation involves the action of $S O(4)$ on $S^{3}$ for each of the $S^{3}$-variables at the given edge.

Proof. For a given face $f \in F$ and a vertex $v \in f_{0}$, the transformation reads

$$
\begin{gathered}
g_{v_{+}(f)}^{(+)} \mapsto h_{v} \cdot g_{v_{+}(f)}^{(+)} \cdot \widetilde{h}_{v}^{-1}, \\
g_{v_{-}(f)}^{(-)} \mapsto h_{v} \cdot g_{v_{-}(f)}^{(-)} \cdot \widetilde{h}_{v}^{-1},
\end{gathered}
$$

so that $\chi^{(j)}\left(g_{v_{+}(f)}^{(+)} \cdot g_{v_{-}(f)}^{(-)}{ }^{-1}\right)$ is invariant.

It is now possible to reconstruct $S O(4)$ parallel transports from the pairs of $S^{3}$-values. Therefore we recall that the action of $S O(4)$ on $S^{3}$ given in Proposition 2.7 is transitive, i.e. for two points $g_{1}, g_{2} \in S^{3}$, there exists a $\left(g_{1}, g_{2}\right) \in S O(4)$ such that

$$
\left(g_{1}, g_{2}\right) * g_{2}=g_{1} \text {. }
$$

Of course, we cannot see elements in the stabilizer of $g_{2}$. Therefore for any $h \in S O(3)$, the action is,

$$
\left(\left(g_{1}, g_{2}\right) \cdot \imath_{g_{2}}(h)\right) * g_{2}=\left(\left(g_{1}, g_{2}\right) \cdot\left(g_{2} h g_{2}^{-1}, h\right)\right) * g_{2}=g_{1} .
$$

We wish to start with an $S O(4)$ gauge theory and then impose the condition that elements from these stabilizers are irrelevant. In other words, we wish to describe an $S O(4)$ gauge theory of which only the action of the parallel transport on $S^{3}$ is physically relevant.

Technically, the key idea is to consider a representation function of $S O(4)$ whose argument is the $S O(4)$ element $\left(g_{1}, g_{2}\right)$ and to average over the stabilizer as parameterized in (4.12b). The result of the averaging is indeed a product of two spherical functions on $S^{3} \cong S U(2)$ with arguments $g_{2}$ and $g_{1}$, respectively. 
Lemma 4.4. Let $t_{m n, p q}^{\left(j j^{\prime *}\right)} \in C_{\mathrm{alg}}(S O(4))$ and $\left(g_{1}, g_{2}\right) \in S O(4)$. Then

$$
\int_{S O(3)} t_{m n, p q}^{\left(j j^{\prime *}\right)}\left(\left(g_{1}, g_{2}\right) \cdot \imath_{g_{2}}(h)\right) d h=\frac{\delta_{j j^{\prime}}}{2 j+1} t_{m n}^{(j)}\left(g_{1}\right) \cdot t_{p q}^{\left(j^{*}\right)}\left(g_{2}\right) .
$$

Remark 4.5. 1. If we integrate (4.13) over $S O(4)$, the right hand side of this equation factorizes into two $S U(2) \cong S^{3}$ integrals.

2. The above lemma crucially makes use of the special relations among $S^{3}, S U(2)$ and $S O(4)$. In particular, in higher dimensional generalizations [19, 29], we would still have $S O(N+1) / S O(N) \cong S^{N}$, but the right hand side of (4.13) would no longer factorize.

3. The averaging over the stabilizer automatically singles out the balanced irreducible representations of $S O(4)$ for which $j=j^{\prime}$.

4. The map

$$
C_{\text {alg }}(S O(4)) \rightarrow C_{\text {alg }}(S O(4)), t_{m n, p q}^{\left(j j^{\prime *}\right)}\left(g_{1}, g_{2}\right) \mapsto \int_{S O(3)} t_{m n, p q}^{\left(j j^{\prime *}\right)}\left(\left(g_{1}, g_{2}\right) \cdot \imath_{g_{2}}(h)\right) d h,
$$

is a projector.

5. In Lemma 4.4, we can replace $S O(4)$ by $\operatorname{Spin}(4) \cong S U(2) \times S U(2)$ in which case the stabilizer is $S U(2)$. The lemma is still satisfied. In particular, each balanced irreducible representation of $\operatorname{Spin}(4)$ is also a representation of $S O(4)$.

Lemma 4.4 suggests that it should be possible to start with an $S O(4)$ lattice gauge theory and then to apply the averaging over the stabilizer in order to restrict the partition function to the balanced irreducible representations and at the same time split the $S O(4)$ integral of the Haar intertwiner into the pair of independent $S^{3}$-integrals in the definition of the Barrett-Crane intertwiner.

In the remainder of this section, we prove that the Barrett-Crane model is indeed obtained from lattice $S O$ (4) $B F$-theory by applying Lemma 4.4 in a suitable manner to the integrand of the partition function. We therefore confirm that the Barrett-Crane model agrees with $S O$ (4) BF-theory subject to constraints [30] whose form we derive at the quantum level.

We wish to apply the averaging procedure of Lemma 4.4 to the $S O(4)$ Haar intertwiner (2.18b) in order to obtain the Barrett-Crane intertwiner (3.6b) together with the balancing (simplicity) condition $j=j^{\prime}$. Obviously, we need one averaging per edge and per face attached to that edge, namely one averaging for each representation function in the Haar intertwiner.

We therefore introduce the exploded configurations of lattice gauge theory which provide several copies of each edge variable so that we have separate variables attaining the same value for all faces attached to a given edge.

Definition 4.6. Let $(V, E, F)$ be a finite 2-complex with cyclic structure.

1. We define the explosion map

$$
X: S O(4)^{E} \mapsto S O(4)^{E \times F},\left\{g_{e}\right\}_{e \in E} \mapsto\left\{g_{(e, f)}\right\}_{(e, f) \in E \times F},
$$

so that $g_{(e, f)}=g_{e}$. The elements of the set $S O(4)^{E \times F}$ are called the exploded configurations. 
2. The simplicity constraint,

$$
P: C_{\mathrm{alg}}\left(S O(4)^{E \times F}\right) \rightarrow C_{\mathrm{alg}}\left(S O(4)^{E \times F}\right),
$$

is defined by specifying its image on a basis of the tensor product $C_{\mathrm{alg}}\left(S O(4)^{E \times F}\right) \cong$ $\bigotimes_{(e, f) \in E \times F} C_{\text {alg }}(S O(4))$, namely for each $(e, f) \in E \times F$,

$$
\begin{aligned}
t_{m n, p q}^{\left(j j^{\prime *}\right)}\left(g_{(e, f)}^{(+)}, g_{(e, f)}^{(-)}\right) & \mapsto \widehat{v}_{j}(2 j+1) \int_{S U(2)} t_{m n, p q}^{\left(j j^{\prime *}\right)}\left(\left(g_{(e, f)}^{(+)}, g_{(e, f)}^{(-)}\right) \cdot \imath g_{(e, f)}^{(-)}(h)\right) d h \\
& =\widehat{v}_{j} \delta_{j j^{\prime}} t_{m n}^{(j)}\left(g_{(e, f)}^{(+)}\right) \cdot t_{p q}^{\left(j^{*}\right)}\left(g_{(e, f)}^{(-)}\right) .
\end{aligned}
$$

3. Given any local Boltzmann weight $w: S O(4) \rightarrow \mathbb{R}$, the exploded Boltzmann weight $\widetilde{W}: S O(4)^{E \times F} \rightarrow \mathbb{R}$, is defined as,

$$
\widetilde{W}\left(\left\{g_{(e, f)}\right\}_{(e, f) \in E \times F}\right):=\prod_{f \in F} w\left(g_{\left(\partial_{1} f, f\right)}^{\varepsilon_{1} f} g_{\left(\partial_{2} f, f\right)}^{\varepsilon_{2} f} \cdots g_{\left(\partial_{N(f)} f, f\right)}^{\varepsilon_{N(f)} f}\right) .
$$

We have absorbed the edge amplitude in its factorized form (4.7) into the definition of the simplicity constraint (4.16).

The partition function of $S O(4)$ lattice gauge theory can, of course, be written in terms of the exploded configurations.

Proposition 4.7. Let $(V, E, F)$ be a finite 2-complex with cyclic structure. The partition function of $S O(4)$ lattice gauge theory on $(V, E, F)$ with Boltzmann weight $w: S O(4) \rightarrow \mathbb{R}$ is equal to

$$
Z=\left(\prod_{e \in E} \int_{S O(4)} d g_{e}\right)(\widetilde{W} \circ X)\left(\left\{g_{e}\right\}_{e \in E}\right) .
$$

We can now state our theorem about the reformulation of the Barrett-Crane model as an $S O(4)$ lattice gauge theory that is subject to the simplicity constraint.

Theorem 4.8 (Gauge theory picture). Let $(V, E, F)$ be a finite 2-complex with cyclic structure. The partition function of $S O(4)$ lattice gauge theory on the exploded configurations, subject to the simplicity constraint (4.16),

$$
Z=\left(\prod_{e \in E} \int_{S O(4)} d g_{e}\right)(P(\widetilde{W}) \circ X)\left(\left\{g_{e}\right\}_{e \in E}\right),
$$

agrees with the Barrett-Crane model (3.10) for face amplitudes,

$$
\widehat{w}_{j}=\int_{S O(4)} \overline{\chi^{\left(j, j^{*}\right)}(g)} w(g) d g,
$$

that are the coefficients of the $S O(4)$ character expansion of the Boltzmann weight for the balanced irreducible representations. The edge amplitudes are of the factorized form (4.7). In particular, for $S O(4)$ lattice $B F$-theory, i.e. $w(g)=\delta(g)$, we obtain the Barrett-Crane model with $\widehat{w}_{j}=(2 j+1)^{2}$. For edge amplitudes (4.7) with coefficients $\widehat{v}_{j}=1 /(2 j+1)$, the simplicity constraint forms a projector. 
Proof. First we consider the Boltzmann weight $\widetilde{W}$ on the exploded configurations and carry out the duality transformation for lattice gauge theory until the analogue of (3.3) for the exploded configurations has been reached. We move all integrals from the Haar intertwiners to the left of the expression and obtain the following integrand,

$$
\begin{aligned}
\widetilde{W}\left(\left\{g_{(e, f)}\right\}_{(e, f) \in E \times F}\right)= & \left(\prod_{\substack { f \in F \\
\begin{subarray}{c}{j_{f}, j_{f}^{\prime} \in \frac{1}{2} \mathbb{N}_{0} \\
j_{f}+j_{f}^{\prime} \in \mathbb{N}_{0}{ f \in F \\
\begin{subarray} { c } { j _ { f } , j _ { f } ^ { \prime } \in \frac { 1 } { 2 } \mathbb { N } _ { 0 } \\
j _ { f } + j _ { f } ^ { \prime } \in \mathbb { N } _ { 0 } } }\end{subarray}}\right)\left(\prod_{f \in F} \widehat{w}_{\left(j_{f} j_{f}^{*}\right)}\right)\left(\prod_{f \in F} \prod_{v \in f_{0}} \sum_{n(f, v)=1}^{2 j_{f}+1} \sum_{m(f, v)=1}^{2 j_{f}^{\prime}+1}\right) \\
\times & \prod_{e \in E}\left(\prod_{f \in e_{+}} t_{n\left(f, \partial_{+} e\right) m\left(f, \partial_{+} e\right), n\left(f, \partial_{-} e\right) m\left(f, \partial_{-} e\right)}^{\left(j_{f} j_{f}^{\prime *}\right)}\left(g_{(e, f)}\right)\right. \\
& \left.\times \prod_{f \in e_{-}} t_{n\left(f, \partial_{+} e\right) m\left(f, \partial_{+} e\right), n\left(f, \partial_{-} e\right) m\left(f, \partial_{-} e\right)}^{\left(j_{f}^{*} j_{f}^{\prime}\right)}\left(g_{(e, f)}\right)\right) .
\end{aligned}
$$

Applying the simplicity constraint (4.16), we find

$$
\begin{aligned}
& (P(\widetilde{W}))\left(\left\{g_{(e, f)}\right\}_{(e, f) \in E \times F}\right) \\
= & \left(\prod_{f \in F} \sum_{j_{f} \in \frac{1}{2} \mathbb{N}_{0}}\right)\left(\prod_{f \in F} \widehat{w}_{\left(j_{f} j_{f}^{*}\right)}\right)\left(\prod_{e \in E} \prod_{f \in \delta e} \widehat{v}_{j_{f}}\right)\left(\prod_{f \in F} \prod_{v \in f_{0}} \sum_{n(f, v)=1}^{2 j_{f}+1} \sum_{m(f, v)=1}^{2 j_{f}+1}\right) \\
\times & \prod_{e \in E}\left(\prod_{f \in e_{+}} t_{n\left(f, \partial_{+} e\right) m\left(f, \partial_{+} e\right)}^{\left(j_{f}\right)}\left(g_{(e, f)}^{(+)}\right) \overline{t_{n\left(f, \partial_{-} e\right) m\left(f, \partial_{-} e\right)}^{\left(j_{f}\right)}\left(g_{(e, f)}^{(-)}\right)}\right. \\
& \left.\prod_{f \in e_{-}} \overline{t_{n\left(f, \partial_{+} e\right) m\left(f, \partial_{+} e\right)}^{\left(j_{f}\right)}\left(g_{(e, f)}^{(+)}\right)} t_{n\left(f, \partial_{-} e\right) m\left(f, \partial_{-}\right)}^{\left(j_{f}\right)}\left(g_{(e, f)}^{(-)}\right)\right),
\end{aligned}
$$

where we write $g_{(e, f)}=\left(g_{(e, f)}^{(+)}, g_{(e, f)}^{(-)}\right)$for the $S O(4)$ variables of the exploded configurations. Setting $g_{(e, f)}=g_{e}$, we can integrate over $S O(4)$ for each edge and obtain two independent $S U(2)$ integrals over $g_{e}^{(+)}$and $g_{e}^{(-)}$per edge. The result agrees with (4.2).

Remark 4.9. 1. In a similar way, we could implement more general edge amplitudes into the simplicity constraint even if they do not factorize. However, the condition that the simplicity constraint forms a projector, singles out a particular edge amplitude. In this case the amplitude factorizes (equation (4.7)), and $\widehat{v}_{j}=1 /(2 j+1)$.

2. In the entire discussion, we can replace $S O(4)$ by $S p i n(4) \cong S U(2) \times S U(2)$ and at the same time $S O(3)$ by $S U(2)$. Passing to the covering does not change any result.

3. The Boltzmann weight (4.8b) in the dual formulation of the Barrett-Crane model is obtained from the Boltzmann weight of lattice gauge theory by applying the simplicity constraint. This means in particular that

$$
\begin{aligned}
& f\left(g_{1}^{(+)}, g_{1}^{(-)}, g_{2}^{(+)}, g_{2}^{(-)}, \ldots, g_{k}^{(+)}, g_{k}^{(-)}\right) \\
= & \int_{S O(3) \times \cdots \times S O(3)} w\left(\left(g_{1}^{(+)}, g_{1}^{(-)}\right) \cdot \imath_{g_{1}^{(-)}}\left(h_{1}\right) \cdots\left(g_{k}^{(+)}, g_{k}^{(-)}\right) \cdot \imath_{g_{k}^{(-)}}\left(h_{k}\right)\right) d h_{1} \cdots d h_{k},
\end{aligned}
$$

where $\left(g_{j}^{(+)}, g_{j}^{(-)}\right) \in S U(2) \times S U(2)$ denote representatives in $S O(4) \cong(S U(2) \times$ $S U(2)) / \mathbb{Z}_{2}$. 
It is no coincidence that the derivation of the group theory picture in the present section closely resembles the reversed direction of the construction of Oriti and Williams [17]. Our result which was motivated by the idea that there is an $S O(4)$ parallel transport associated to the tetrahedra (dual edges), turns out to agree with a 'trivial gluing' in [17]. In [17], a certain prescription for the gluing of four-simplices is employed in order to extend the geometrical constraints on a single four-simplex 14 to the entire triangulation. Different ways of imposing the constraints in the gluing procedure would give rise to different edge amplitudes [28]. In our formulation, the edge amplitude $\widehat{v}_{j}$ in (4.16) looks a bit artificial. For our choice of the constraint, only one amplitude seems to be natural, namely the case in which $P$ is a projector. In order to implement the constraints differently in the gluing procedure, one would have to change the constraint $P$ and perhaps also the combinatorics of the explosion map and would then recover a preferred edge amplitude from the requirement that $P$ forms a projector. The results of [17] demonstrate that there exist alternative constraint maps $P$ that have a particular geometric interpretation and that give rise to different edge amplitudes. Further work on these matters is in progress [28].

\subsection{Regularizing the Boltzmann weight}

The reformulation of the Barrett-Crane model in terms of continuous variables presented in Theorem 4.1, offers a framework in which one can study the ground state of the model and small fluctuations around it, and which is also well suited for numerical studies. In this section, we sketch some central ideas and also comment on the question of which amplitudes $\widehat{w}_{j}$ and $\mathcal{A}^{(e)}$ are admissible in the partition function (3.10).

The amplitudes $\widehat{w}_{j}$ and $\mathcal{A}^{(e)}$ are not fixed from geometric considerations 䟣. The simplest choice is,

$$
\widehat{w}_{j}=\operatorname{dim} V_{j} \otimes V_{j}^{*}=(2 j+1)^{2}, \quad \mathcal{A}^{(e)}=1 .
$$

In this case the Boltzmann weight (4.8b) behaves similarly to a $\delta$-function whose arguments are the products $g_{\ell+1}^{(+)} \cdot g_{\ell}^{(-)^{-1}}$. If the two $S^{3}$-values of each pair agree, the weight (4.8b) diverges because $\lim _{\varphi \rightarrow 0} \chi^{(j)}(\varphi)=2 j+1$. On the other hand, a simple numerical calculation suggests that the weight is very small (i.e. grows with $j$ only much more slowly than the peak at $\varphi=0)$ whenever there is a pair of $S^{3}$-variables whose values do not agree.

In order to obtain a first intuition, consider Figure 8 and assume that the Boltzmann weight vanishes whenever two $S^{3}$-variables (dots) that form a pair in one of the characters (pair of solid lines) are not aligned. According to the structure of double lines in Figure \&(b), there exists a vanishing weight unless all five $S^{3}$-values agree. The four-simplices would therefore be rigid, and the hyperplanes of all five tetrahedra in the boundary of a given foursimplex would have to be aligned. This is geometrically a very degenerate configuration in which the four-simplex has zero four-volume. In addition, there is no relation between the $S^{3}$-values assigned to different four-simplices. If we transit from one four-simplex along a tetrahedron to a neighbouring four-simplex and imagine that the change in $S^{3}$-value is given by a parallel transport along the (edge dual to the) tetrahedron, then there is no restriction on that parallel transport.

However, these arguments are not quite correct because we did not precisely quantify the value of the total Boltzmann weight if we multiply very large (if $\varphi=0$ ) and very small (if some other $\varphi \neq 0$ ) numbers. We therefore have to regularize the weight function. This can 
be done, for example, by a non-trivial edge amplitude $\mathcal{A}^{(e)}$ or by introducing a one parameter family of functions that tends to the degenerate face amplitude in a suitable 'limit'. Obvious choices of amplitudes are the following.

Lemma 4.10. Let $\widehat{w}_{j} \in \mathbb{C}$ denote the face amplitudes, let $\widehat{v}_{j} \in \mathbb{C}$ define the factorized edge amplitude (4.7), and

$$
f_{k}\left(\varphi_{0}, \ldots, \varphi_{k}\right)=\sum_{j \in \frac{1}{2} \mathbb{N}_{0}} \widehat{w}_{j} \widehat{v}_{j}^{k} \prod_{\ell=1}^{k} \frac{\sin \left((2 j+1) \varphi_{\ell}\right)}{\sin \left(\varphi_{\ell}\right)}
$$

denote the Boltzmann weight (4.8b) for a $k$-gon, expressed in terms of the dihedral angles $\varphi_{\ell} \in[-\pi, \pi)$ on $S^{3}$.

1. For $\widehat{w}_{j}=(2 j+1)^{2}$ and $\widehat{v}_{j}=1$ or $\widehat{v}_{j}=1 /(2 j+1)$, the series does not converge if all $\varphi_{j}=0$.

2. If $\widehat{w}_{j}=(2 j+1)^{2}$ and $\widehat{v}_{j}=1 /(2 j+1)^{2}$, then $f_{k}, k \geq 2$, converges pointwise for all $\varphi_{\ell}$ because the characters are bounded, $|\sin (n \varphi) / \sin (\varphi)| \leq n$ for all $\varphi$.

3. If $\widehat{w}_{j}=(2 j+1)^{2} \exp \left(-\frac{j(j+1)}{\beta}\right)$ and $\widehat{v}_{j}=1$ or $\widehat{v}_{j}=1 /(2 j+1)$, then $f_{k}$ converges pointwise for all $\varphi_{\ell}$ and any $\beta>0$.

Furthermore, a simple numerical calculation suggests that $f_{k}$ is strictly positive only in case (3) provided that $\widehat{v}_{j}=1 /(2 j+1)$, and that in the cases (2) and (3), the function $f_{k}$ has a unique maximum when all $\varphi_{\ell}=0$. Both choices (2) and (3) yield a non-trivial shape of the function $f_{k}$. Therefore the four-simplices will be not exactly rigid and degenerate, and there will be a non-trivial interaction between the four-simplices mediated by the Boltzmann weights for each triangle.

In order to obtain a well-defined partition function (4.8a), it is sufficient that the integrand is an $L^{2}$ function of the $S U(2)$-variables. However, for numerical studies and also for an intuitive understanding, it is useful to have a pointwise convergent series (4.25) so that we can always compare the function values for different angles $\varphi_{j}$.

If we insist on a strictly positive and regular Boltzmann weight function and on the projector property of the simplicity constraint, choice $(3)$ with $\widehat{v}_{j}=1 /(2 j+1)$ for which the $\widehat{w}_{j}$ are given by the character expansion coefficients (4.20) of the $S O(4)$ heat kernel action (for one of the Casimir operators) [25, 26], deserves to be studied in greater detail. In this case, there exists a limit $\beta \rightarrow \infty$ in which the Boltzmann weight tends to the degenerate weight $\widehat{w}_{j}=(2 j+1)^{2}$ of the Barrett-Crane model.

Choice (3) therefore defines a generalized Barrett-Crane model with a bare 'inverse temperature' $\beta=1 / g_{0}^{2}$ or with a bare 'coupling constant' $g_{0}$. This regularized model provides a framework in which one could study the classical ground state, i.e. the configuration of maximal probability, and small fluctuations around it. The Boltzmann weight (4.8b) has a non-trivial shape, and it makes sense to ask the question of whether the interaction terms for the triangles can be responsible for long range correlations. A perturbative analysis would correspond to an asymptotic expansion around $\beta \rightarrow \infty$ which is precisely the interesting case.

In the model with generic $\beta$, the configurations of the spin foam formulation of the BarrettCrane model correspond to the coefficients of the strong coupling expansion, i.e. of an expansion for small $\beta$, similarly to the situation in lattice gauge theory [8, 9]. At strong bare coupling, the configurations for small representations dominate. 
At this point, some warnings are appropriate. The reformulation of the Barrett-Crane model (4.8a might exhibit significant non-perturbative effects. For all choices of amplitudes $\widehat{w}_{j}$ and $\widehat{v}_{j}$ discussed above, the classical ground state, i.e. the state of maximal probability, is the configuration in which all tetrahedra of a given four-simplex are aligned. This configuration is geometrically degenerate and spans only a zero four-volume, so that one would need quantum effects in order to regularize the degeneracy and to provide a proper four-volume. This in turn means that the typical configurations one would find in the path integral, are 'far away' from the classical ground state. Confinement in pure $S U(3)$ gauge theory is a famous example for such a non-classical behaviour. In this case, perturbation theory cannot be expected to describe the quantum system correctly because it is an expansion around the wrong vacuum.

The new parameter $\beta$ and maybe the freedom to choose different edge amplitudes, also raise the question of whether and how we have to renormalize the model. From the above considerations, one might conjecture that the Barrett-Crane model had to be renormalized along the lines of lattice gauge theory.

The choice of a triangulation or of a dual 2-complex for a given smooth manifold would correspond to a choice of coordinates, and the triangulation had to be 'fine' enough, i.e. had to contain sufficiently many simplices, for the aspects we wish to study. An interesting question is certainly whether there exist long range correlations which would arise from local degrees of freedom of the model and correspond in some sense to the Euclidean analogue of the graviton. Long range is here referred to in a combinatorial sense, i.e. variables that are separated by many simplices should still be correlated. Provided that such long range effective degrees of freedom exist, several scenarios are conceivable.

First, we might have to tune or fine-tune $\beta$ or the edge amplitude in order to obtain an infinite or at least a very large correlation length. Alternatively the model could be, at least for some range of parameters, in a Coulomb like phase and exhibit correlations that decay with the reciprocal distance (again in the combinatorial sense). This would provide a scenario in which there are always long range correlations and which is largely insensitive to the choice of parameters.

After one has finally understood the spectrum of correlation lengths of the model in a combinatorial sense of 'length', one would have to reconstruct metric information from the data provided by the model so that, say in a suitable 'classical' limit, the combinatorial correlations could be interpreted as correlation functions with respect to a metric distance. It is an interesting question how a fundamental length scale might arise in such an approach.

Finally, we mention that two versions of the Barrett-Crane model, [15] with normalized intertwiner, and [16, 17], have edge amplitudes that do not factorize. In this case, the dual model (4.6) has a non-local Boltzmann weight, and the classical ground state is no longer obvious and might even have better properties. This is a second possibility, besides quantum effects, how one could avoid the degenerate ground state. A more detailed study of the possible edge amplitudes of the dual model is in preparation.

\section{Conclusion}

We have presented a reformulation of the Euclidean Barrett-Crane model in terms of dual $S^{3}$-variables. The new variables have a geometric interpretation and can be understood as variables conjugate to the quantized areas. 
The fact that the dual model can be phrased in terms of continuous variables, prepares the ground for the application of a variety of analytical and numerical techniques. Interesting are in particular the question of how the degenerate classical ground state can be regularized, maybe by quantum effects, maybe by a non-local Boltzmann weight for the dual model or maybe only if we introduce a cosmological constant or couple gravity to matter. Also important is the question of which correlations are relevant in the dual model.

The possible choices of edge amplitudes and regularization parameters $\beta$ raise the question of whether and how one has to renormalize the theory. On the one hand, the model is not immediately triangulation independent, so that some refinement or sum over triangulations will be necessary. On the other hand, the result (however this will be achieved) is expected to be background independent. This requires an entirely new interpretation of the renormalization procedure as opposed to what is familiar from the context of lattice gauge theory and Statistical Mechanics. We are confident that the reformulation presented here offers a framework in which these questions can be approached.

\section{Acknowledgements}

The author is grateful to Emmanuel College, Cambridge, for a Research Fellowship. I would like to thank John Barrett, Mátyás Karádi, Alan Macfarlane, Robert Oeckl, Daniele Oriti, Nuno Romão, Ruth Williams and Toby Wiseman for many valuable discussions, for comments on the manuscript and on relevant literature.

\section{References}

[1] J. C. BAEz: Spin, loops, knots and gauge fields. In Knots and Quantum Gravity, ed. J. C. Baez. Oxford University Press, Oxford, 1994.

[2] M. P. Reisenberger: World sheet formulation of gauge theories and gravity. Preprint gr-qc/9412035.

[3] M. P. Reisenberger and C. Rovelli: 'Sum over surfaces' form of loop quantum gravity. Phys. Rev. D 56 (1997) 3490-3508, gr-qc/9612035.

[4] J. W. Barrett and L. Crane: Relativistic spin networks and quantum gravity. J. Math. Phys. 39, No. 6 (1998) 3296-3302, gr-qc/9709028.

[5] J. C. BAez: Spin foam models. Class. Quant. Grav. 15, No. 7 (1998) 1827-1858, gr-qc/9709052.

[6] V. G. Turaev and O. Y. Viro: State sum invariants of 3-manifolds and quantum 6j-symbols. Topology 31, No. 4 (1992) 865-902.

[7] L. Crane, L. H. Kauffmann and D. N. Yetter: State-sum invariants of 4manifolds. J. Knot Th. Ramif. 6, No. 2 (1997) 177-234, hep-th/9409167.

[8] R. Oeckl and H. Pfeiffer: The dual of pure non-Abelian lattice gauge theory as a spin foam model. Nucl. Phys. B 598, No. 1-2 (2001) 400-426, hep-th/0008095. 
[9] H. Pfeiffer and R. Oeckl: The dual of non-Abelian Lattice Gauge Theory. To be published in the proceedings of Lattice 2001 (2002). Preprint hep-th/0110034.

[10] H. Pfeiffer: Four-dimensional Lattice Gauge Theory with ribbon categories and the Crane-Yetter state sum. J. Math. Phys. 42, No. 11 (2001) 5272-5305, hep-th/0106029.

[11] R. OEckL: Generalized lattice gauge theory, spin foams and state sum invariants (2001). Preprint hep-th/0110259.

[12] J. C. BAEz: An introduction to spin foam models of $B F$ theory and quantum gravity. In Geometry and quantum physics, Lecture Notes in Physics 543. Springer, Berlin, 2000, pp. 25-93, gr-qc/9905087.

[13] D. OrITI: Space-time geometry from algebra: Spin Foam Models for nonperturbative quantum gravity. Rep. Prog. Phys. 64 (2001) 1703-1756, gr-qc/0106091.

[14] J. C. BAez and J. W. Barrett: The quantum tetrahedron in 3 and 4 dimensions. Adv. Th. Math. Phys. 3, No. 4 (1999) 815-850, gr-qc/9903060.

[15] R. De Pietri, L. Freidel, K. Krasnov and C. Rovelli: Barrett-Crane model from a Boulatov-Ooguri field theory over a homogeneous space. Nucl. Phys. B 574, No. 3 (2000) 785-806, hep-th/9907154.

[16] A. Perez and C. Rovelli: A spin foam model without bubble divergences. Nucl. Phys. B 599, No. 1,2 (2001) 255-282, gr-qc/0006107.

[17] D. Oriti and R. M. Williams: Gluing 4-simplices: a derivation of the Barrett-Crane spin foam model for Euclidean quantum gravity. Phys. Rev. D 63 (2001) 024022, gr-qc/0010031.

[18] J. W. Barrett: The classical evaluation of relativistic spin networks. Adv. Th. Math. Phys. 2, No. 3 (1998) 593-600, math/9803063.

[19] L. Freidel and K. Krasnov: Simple spin networks as Feynman diagrams. J. Math. Phys. 41, No. 4 (2000) 1681-1690, hep-th/9903192.

[20] H. Ooguri: Topological lattice models in four dimensions. Mod. Phys. Lett. A 7, No. 30 (1992) 2799-2810.

[21] N. Kawamoto, N. Sato and Y. Uchida: 4-dimensional BF gravity on the lattice. Nucl. Phys. B 574 (2000) 809-848, hep-th/9911228.

[22] J. C. Baez and J. D. Christensen: Positivity of spin foam amplitudes (2001). Preprint gr-qc/0110044.

[23] N. Ja. Vilenkin and A. U. Klimyk: Representations of Lie groups and special functions - Class I representations, special functions and integral transforms, volume 2. Kluwer Academic Publishers, Doordrecht, 1993.

[24] R. Carter, G. Segal and I. Macdonald: Lectures on Lie groups and Lie algebras. London Mathematical Society Student Texts 32. Cambridge University Press, Cambridge, 1995. 
[25] H. J. Rothe: Lattice Gauge Theories - An Introduction. World Scientific, Singapore, 1992.

[26] I. Montvay and G. Münster: Quantum fields on a lattice. Cambridge University Press, Cambridge, 1994.

[27] M. P. Reisenberger: On relativistic spin network vertices. J. Math. Phys. 40, No. 4 (1999) 2046-2054, gr-qc/9809067.

[28] D. ORITI: In preparation.

[29] L. Freidel, K. Krasnov and R. Puzio: BF description of higher-dimensional gravity theories. Adv. Th. Math. Phys. 3 (1999) 1289-1324, hep-th/9901069.

[30] R. De Pietri and L. Freidel: so(4) Plebaǹski action and relativistic spin-foam model. Class. Quant. Grav. 16 (1999) 2187-2196, gr-qc/9804071. 